\title{
Comparação linguística e perfilação gramatical sistêmica em um corpus combinado
}

\section{Linguistic comparison and grammatical systemic profiling in a bidirectional parallel corpus}

Francieli Silvéria Oliveira

Universidade Federal de Ouro Preto - UFOP, Minas Gerais.

franciellysilveria@hotmail.com

Resumo: Com base nos pressupostos da Linguística de Corpus (BERBER SARDINHA, 2000; VIANA, 2011), este trabalho investiga a organização gramatical e semântica de um corpus combinado de manual de instrução no par linguístico inglês / português brasileiro, objetivando apresentar a variação linguística característica desse registro, bem como comparar as línguas e descrever a produção textual de significado da tradução técnica. Pesquisas anteriores apresentam que é possível estudar os registros de uma língua por meio da análise de corpus (BIBER, 2010). Figueredo (2014) propõe o uso da perfilação gramatical sistêmica para encontrar padrões gramaticais. Mediante esses conceitos, os resultados apresentaram que o manual de instrução tem como padrão linguístico as funções semânticas 'explicar', 'comandar', 'classificar' e 'introduzir' com suas respectivas funções gramaticais. Com relação à sua tradução técnica, conclui-se que ela é constituída de textos híbridos e multilíngues que, para significar, utilizam-se do pareamento do texto fonte, da língua alvo e de novos significados.

Palavras-chave: comparação linguística; perfilação gramatical sistêmica; metodologia baseada em corpus; tradução técnica.

Abstract: This paper investigates the grammatical and semantic organization of a bidirectional parallel corpus of instruction manuals based on Corpus Linguistics. It aims at presenting the linguistic variation of this register as well as describing text production of technical translations. Previous researches show it is possible to study register through corpus analysis (BIBER, 2010). 
Figueredo (2014) suggests using grammar systemic profiling to find grammar patterns. Results show that instruction manuals present linguistic patterns of semantic functions 'expounding', 'commanding', 'classifying' and 'introducing', along with their respective grammar functions. Regarding technical translations, translated manuals are shown to be hybrids and multilingual texts, which mean they use coupling of source text, target language, and new meanings.

Keywords: linguistic comparison; grammatical systemic profiling; corpusbased methodology; technical translation.

Recebido em: 31 de julho de 2015. Aprovado em: 29 de setembro de 2015.

\section{Introdução}

Com base nos pressupostos da Linguística de Corpus (BERBER SARDINHA, 2000; BIBER, 2010; VIANA, 2011), o presente artigo investiga o funcionamento gramatical e semântico de um corpus combinado (STEINER, 2001; NUNES, 2013) do Manual de Instrução, procurando explicar como se dá a construção de significados desse registro e sua generalização para a Tradução Técnica (AZENHA, 1995).

Tendo como objeto de estudo a produção textual de significado (HALLIDAY, 1994), mais especificamente, a 'dinâmica textual' (LEMKE, 1993), objetiva-se apresentar os padrões gramaticais e semânticos que compõem os manuais de instrução no par linguístico inglês / português brasileiro, por meio da descrição de seus perfis sistêmicos (HALLIDAY, 1978; FIGUEREDO, 2014) como forma de compreender a variação linguística que constrói o texto técnico e a tradução técnica. Complementarmente, visa-se construir um modelo multilíngue que contenha os padrões encontrados no corpus e que seja capaz de explicar a sua dinâmica textual.

A Linguística de Corpus (LC) e Linguística Sistêmico-Funcional (LSF) constituem base teórica e metodológica desta pesquisa. A LC exerce esse papel por investigar a língua empiricamente, utilizando, para 
isso, textos produzidos por falantes de forma espontânea (VIANA, 2011) e que, por isso, compõem o corpus de investigação. Por essa razão, Biber (2010, p. 241) propõe que o estudo dos registros de uma língua deve ser pautado na análise de corpus, pois ele aponta o funcionamento textual dos registros, como também apresenta seus padrões linguísticos; padrões esses que convencionalmente se associam à sua função cultural. Nesse sentido, os padrões gramaticais e semânticos do manual determinam o que é esse registro: uma macro-onda de significado que capacita o falante a desenvolver uma atividade, instruindo-o de forma a não regular seu comportamento.

Os manuais de instrução aqui utilizados são denominados textos técnicos por apresentarem uma abordagem diferente dos textos expositivos e por se tratarem de uma área específica (AZENHA, 1995). $\mathrm{Na}$ Tradução Técnica, Azenha (1995, p. 141) afirma que essa área de estudo apresenta problemas, tais como: lexical-terminológicos, grafofonológicos, sintáticos, terminológicos, morfossintáticos, semânticos e receptivos. Dessa maneira, para compreender as relações envolvidas nesse tipo de tradução, faz-se necessária uma reflexão mais sistemática sobre o texto. Para essa reflexão, Catford (1965, p. 35) afirma que a comparação linguística em tradução deve se pautar por uma teoria que consiga descrever tanto a língua fonte quanto a língua alvo. Devido a seu potencial de impacto nesse aspecto (MATTHIESSEN, 2001), a Linguística Sistêmico-Funcional é aqui adotada. A LSF (HALLIDAY, 2013), por ser uma teoria abrangente, consegue descrever e analisar a língua em todos os seus estratos, tais como: a gramática, a semântica e o contexto.

Como forma de cumprir o objetivo desta pesquisa, foi compilado um corpus combinado (NUNES, 2013) com quatro manuais de instrução. Do notebook Macbookpro Retina 13 inch - cf. website "Apple" - coletaram-se o texto original em inglês e sua tradução para o português brasileiro. Da Panela de Pressão Solar Tramontina - cf.

\footnotetext{
${ }^{1} \mathrm{Cf}$.

$<$ http://manuals.info.apple.com/MANUALS/1000/MA1663/en_US/macb ook

pro_retina-13-inch-late-2013_qs.pdf>.
} 
website "Tramontina"2 - coletaram-se o texto original em português brasileiro e a tradução para o inglês.

Para a análise do corpus, os sistemas gramaticais TRANSITIVIDADE, MODO e TEMA (HALLIDAY; MATTHIESSEN, 2014) foram utilizados para compreender a produção textual de significado e determinar os padrões gramaticais e semânticos do manual. Mais especificamente para comparar os textos nos pares original / tradução e original / original, a fim de responder como se caracteriza a tradução técnica desse registro: um texto multilíngue que, para significar, utiliza-se do pareamento do texto fonte, da língua alvo e de novos significados.

Com base na metodologia de perfilação gramatical (FIGUEREDO, 2014), o artigo apresenta como resultado o perfil gramatical sistêmico do corpus combinado de manuais. Esse perfil mostra as escolhas gramaticais e semânticas mais recorrentes dos manuais de instrução, apresentando, assim, seu funcionamento, além de converter "funções gramaticais em "tokens gramaticais" (FIGUEREDO, 2014, p. 18), ampliando as buscas de padrões por meio da metodologia de corpus.

Ao final, este artigo demonstra como o arcabouço da LC com a LSF possibilitam apresentar o funcionamento da produção textual de significado do registro: manual de instrução, assim como permite compreender de que modo a tradução técnica do manual de instrução é construída mediante a comparação da variação linguística do corpus combinado.

\section{A Linguística de Corpus na comparação linguística e perfilação sistêmica}

Tendo como principal objetivo estudar a língua em seu funcionamento por meio de dados linguísticos reais, produzidos por

\footnotetext{
${ }^{2} \mathrm{Cf}$.

$<$ http://suipnovo.tecnologia.ws/public/upload/product/62516223/625162 22FLM001>.
} 
falantes de forma espontânea, a LC ocupa-se da compilação e análise do corpus com base em padrões emergentes do uso da língua. Sinclair (1997, p. 31) afirma que "a língua não pode ser inventada; mas apenas capturada". Levando isso em consideração e tomando como base os pressupostos metodológicos da LC, o corpus de pesquisa deve ser coletado de forma criteriosa para servir de base para o estudo da língua e da variedade linguística em foco.

Desse modo, Berber Sardinha (2000, p. 338) coloca como condição fundamental para a pesquisa a necessidade do corpus possuir dados autênticos. O autor observa que o corpus deve ser compilado segundo o propósito da pesquisa, que seus dados devem ser criteriosamente coletados, que devem representar a língua, além de serem legíveis por meio do computador.

Como a LC apresenta métodos para tratar dados linguísticos (VIANA, 2011), ela pode ser usada em vários campos dos estudos da linguagem, como o estudo do léxico, da gramática, no ensino de línguas, no estudo dos registros, nos estudos da tradução, entre outros. No caso deste artigo, a LC foi utilizada no estudo do registro 'manual de instrução' e na sua tradução, por meio da comparação linguística (CATFORD, 1965; KRZESZOWSKI, 1990) e da perfilação sistêmica (FIGUEREDO, 2014).

\subsection{Comparação linguística e estudos da tradução}

Nos estudos da tradução, a LC cumpre papel fundamental de base metodológica pelo fato de trabalhar com a língua em uso (BERBER SARDINHA, 2002), uma vez que o corpus compilado e construído com o propósito de comparar as línguas contribui para a realização de uma pesquisa mais eficaz e replicável (VIANA, 2011).

Nos estudos da tradução, a comparação entre línguas é uma forma de fazer a análise textual das traduções e descobrir os processos linguísticos que as envolvem. Halliday et al. (1964) afirmam que todas as línguas são sistematicamente comparáveis, uma vez que elas possuam categorias teóricas que consigam descrevê-las. Diante disso, Catford (1965) apresenta dois tipos de comparação linguística: a equivalência textual e a correspondência formal. A equivalência textual ocorre quando 
uma porção de texto na língua fonte, por permutação, é equivalente na língua alvo. A correspondência formal ocorre quando se pode apontar que uma categoria teórica do texto alvo possui uma categoria teórica correspondente na língua fonte. Como forma de ampliar o estudo de Catford, Krzeszowski (1990) afirma que as línguas são estruturalmente comparáveis, na medida que são estruturalmente semelhantes. Neste artigo, as categorias utilizadas na análise do corpus de manuais de instrução descrevem o inglês e o português brasileiro (FIGUEREDO, 2011; FIGUEREDO, 2015).

O manual de instrução aqui analisado é um texto técnico, portanto, a sua tradução, que também trata de uma área específica, é uma tradução técnica. A respeito desse campo da tradução, Azenha (1995) propõe que todos os níveis de hierarquia linguística são importantes para o estudo e a tradução do texto técnico, sendo assim, necessária uma reflexão mais sistemática sobre esse tipo de tradução. Para essa reflexão, a LSF foi utilizada na análise do corpus.

\subsection{Perfilação gramatical sistêmica}

A perfilação gramatical é uma metodologia baseada nos pressupostos da LC que permite a identificação de padrões e a análise de corpora para a investigação de como as funções gramaticais constroem significado na dinâmica textual (FIGUEREDO, 2014, p. 17). Essa metodologia contribui para os estudos da LC por possibilitar a conversão de categorias gramaticais em "tokens gramaticais", permitindo, assim, o processamento da gramática automaticamente.

Uma vez que língua é compreendida pela LC como probabilidade, o estudo dos padrões de uso recorrentes, como também a ocorrência da cosseleção de itens (VIANA, 2011, p. 39) se torna a forma mais eficaz de investigação. A perfilação gramatical segue esses preceitos, analisando as ocorrências gramaticais oracionais e detectando padrões nas cosseleções gramaticais.

Por conseguinte, a perfilação gramatical também contribui na busca de padrões em corpora, por meio da qual é possível ainda identificar padrões gramaticais relacionados ao estudo dos registros. Biber (2010, p. 246) aponta que o estudo dos registros de uma língua 
deve ser embasado em dados de corpus, dados esses que apontam a variação linguística característica dos registros. É mediante a frequência no corpus que os padrões linguísticos podem ser encontrados (BIBER et al., 2004, p. 376).

A perfilação gramatical se vale da LSF por ela constituir-se de uma teoria que abrange todos os estratos linguísticos e por possuir, em cada estrato, categorias dispostas em rede de sistemas para analisar e descrever a língua (cf. HALLIDAY; MATTHIESSEN, 2014; entre outros). Pela LSF, é possível compreender como as escolhas linguísticas estão dispostas no corpus e como elas, pelas suas cosseleções, constroem o significado de cada texto.

\section{Metodologia}

Neste artigo, é apresentada a construção da tradução técnica do corpus de manual de instrução no par linguístico inglês e português brasileiro tendo como base a LC (BERBER SARDINHA, 2000; BIBER, 2010; VIANA, 2011) e a LSF (HALLIDAY, 2002; MARTIN; ROSE, 2007; FIGUEREDO, 2014), como também o perfil sistêmico do corpus combinado. Para tanto, a seguir serão apresentados os procedimentos aqui utilizados.

\subsection{Corpus}

Para analisar o funcionamento gramatical dos manuais, foi compilado um corpus combinado (STEINER, 2001; KENNING, 2010), definido por Nunes (2013, p. 7) como

um córpus bidirecional no qual um conjunto de textos originais em uma língua $\mathrm{A}$ são traduzidos para uma língua $\mathrm{B}$ e originais na língua $\mathrm{B}$ são traduzidos para a língua $\mathrm{A}$, de forma que seja possível combinar todas as perspectivas paralelas e comparáveis para análise de dados. 
No corpus combinado deste artigo, a língua A mencionada por Nunes se refere ao inglês e a língua $\mathrm{B}$ ao português brasileiro. Além disso, a respeito do número de tokens coletados para cada texto, este trabalho segue a proposta metodológica de Biber (1990), que aponta o número mínimo de 1.000 tokens para representar uma amostra autêntica da língua utilizada e para analisar os padrões de frequência de um corpus.

Dessa forma, foram coletados quatro manuais de instrução para o corpus combinado de 1.000 tokens cada um. Em inglês original e tradução para o português brasileiro, foram coletados no website "Apple" os manuais do Macbookpro retina 13 inch, e em português brasileiro original e tradução para o inglês foram coletados no website "Tramontina" os manuais da panela de pressão Solar Tramontina.

Os textos foram compilados de acordo com a tipologia da língua no contexto de cultura (URE, 1989; MATTHIESSEN et al., 2008) e tiveram como base os oito processos sociossemióticos: explicar, relatar, recriar, compartilhar, fazer, recomendar, capacitar e explorar, e os quatro modos de produção: escrito / monólogo, escrito / diálogo, falado / monólogo e falado / diálogo.

Halliday e Matthiessen (2014, p. 35) dividem esses oito processos sociossemióticos em dois grupos, o primeiro em fazer e o segundo em significar: (1) processo sociossemiótico 'fazer': semiotiza significados relacionados com o comportamento e a ação humanos; nesse sentido, a língua é usada para facilitar a execução de uma atividade; (2) 'significar' abarca os demais processos sociossemióticos: caracteriza-se por constituir uma situação na qual os significados são criados. A Figura 1 apresenta os processos sociossemióticos: 
Figura 1 - Processos sociossemióticos e os modos de produção com exemplos

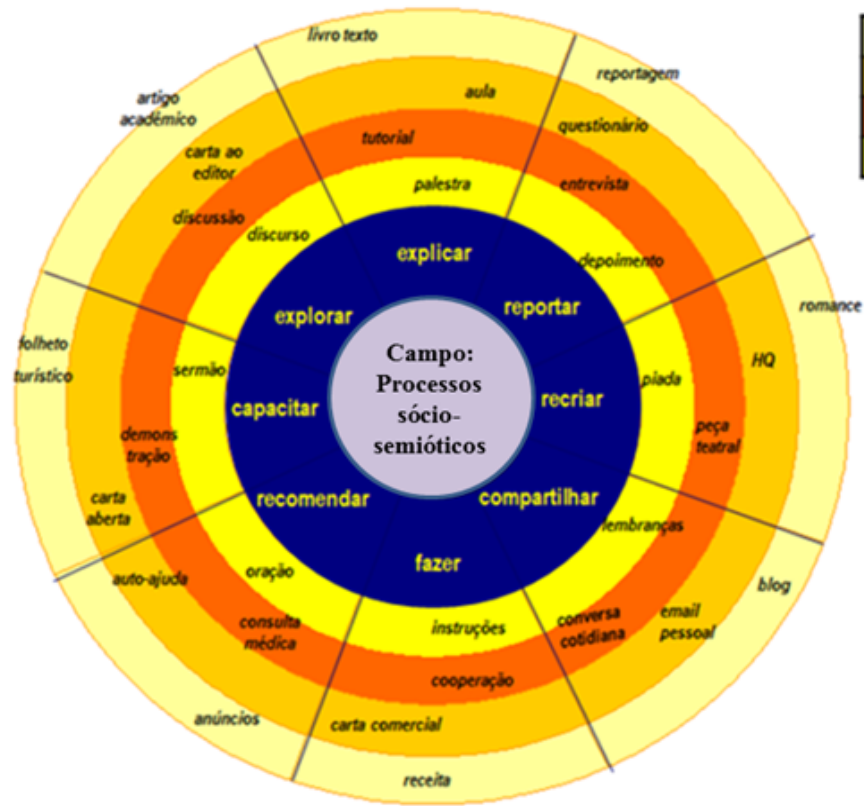

\begin{tabular}{|l|l|}
\hline escrito & monólogo \\
\hline escrito & diálogo \\
\hline falado & diálogo \\
\hline falado & monólogo \\
\hline
\end{tabular}

Fonte: Adaptado de Matthiessen et al., 2008.

Os textos do corpus deste artigo estão localizados no processo sociossemiótico 'capacitar' e no modo de produção escrito monólogo. Textos pertencentes a esse processo sociossemiótico podem conter procedimentos simples, como também procedimentos altamente técnicos (HALLIDAY; MATTHIESSEN, 2014).

Os manuais de instrução compilados para esta pesquisa têm como objetivo auxiliar o falante a usar adequadamente o aparelho em questão; no caso, o notebook da marca Apple e a panela de pressão Solar da Tramontina.

Após a coleta do corpus, os manuais de instrução foram etiquetados e armazenados. Para o etiquetamento e armazenamento, este trabalho teve como base as regras do Catálogo da Língua Brasileira (CALIBRA). 
3.2 Metodologia de Análise

Cada texto do corpus combinado - após coleta nos websites e subsequente conversão para formato txt - foi transferido para a ferramenta computacional UAM CorpusTool ${ }^{\circ}$ (O’DONNELL, 2008). A análise teve como base os sistemas gramaticais TRANSITIVIDADE, MODO e TEMA (HALLIDAY; MATTHIESSEN, 2014). Como o corpus desta pesquisa é composto por duas línguas, foram utilizadas categorias do ambiente multilíngues (FIGUEREDO, 2015), dessa maneira, as categorias descritas a seguir representam aquelas utilizadas na análise do corpus.

O sistema de TRANSITIVIDADE é responsável por apresentar a língua em ação, por representar gramaticalmente o que acontece no mundo. Para a análise, utilizaram-se suas funções de Processos Material, Mental, Relacional, Verbal e Comportamental (HALLIDAY; MATTHIESSEN, 2014).

A metafunção interpessoal é responsável por apresentar o nível de relação existente entre os interlocutores de um texto. Essa categoria é representada pelo sistema de MODO que possui as funções de Imperativo: Jussivo, Imperativo: Sugestivo, Indicativo: Declarativo, Indicativo: Interrogativo: Polar e Indicativo: Interrogativo: Elemental (FIGUEREDO, 2011).

O sistema de TEMA (MARTIN; ROSE, 2007) gera as funções: Default, Tema Elemental, Ângulo: Fonte, Ângulo: Ponto de Vista, Perspectiva e Intensivo.

Posterior à análise da TRANSITIVIDADE, MODO e TEMA, para compreender as fases do discurso que compõem o manual, também foi feita a análise do corpus pelo viés das ondas de informação. Para tanto, foi incluído na análise do perfil metafuncional o sistema de MENSAGEM (cf. MARTIN; ROSE, 2007). As ondas de informação representam as fases do discurso em que os significados são reunidos para apresentar um significado maior.

3.3 Metodologia de perfilação sistêmica 
Para a criação do perfil sistêmico do corpus, foram necessários procedimentos para visualizar a disposição dos significados de cada manual e, assim, realizar a perfilação. Para isso, as categorias da LSF foram colocadas no espaço gramatical segundo o mapa topológico de cada metafunção, estabelecendo-se, assim, suas distâncias relativas (FIGUEREDO, 2014).

As distâncias topológicas, então representadas por distâncias topológicas gramaticais, formam a base da descrição das orações de cada texto. Para exemplificar, o Quadro $1^{3}$ apresenta um exemplo da análise das distâncias topológicas, aqui descritas como um código numérico.

Quadro 1 - Exemplo de descrição das distâncias topológicas no espaço gramatical expressa em código numérico

\begin{tabular}{l|c|c|c|c}
\hline ORAÇÃO & Transitividade (x) & Modo (y) & Tema (z) & $\begin{array}{c}\text { Ondas de } \\
\text { Informação }\end{array}$ \\
\hline CAP_01_01 & 14 & 20 & 10 & $1^{\mathrm{a}}$ \\
CAP_01_02 & 14 & 20 & 10 & \\
CAP_01_03 & 39 & 20 & 10 & \\
CAP_01_04 & 14 & 20 & 45 & $2^{\mathrm{a}}$ \\
CAP_01_05 & 39 & 11 & 10 & \\
CAP_01_06 & 16 & 20 & 10 & \\
CAP_01_07 & 14 & 11 & 10 & \\
\hline
\end{tabular}

O Quadro 1 aponta o número de cada oração composto pelo código da etiqueta do texto (por exemplo: CAP_01) com o número referente à ordem de cada oração (por exemplo: $\mathrm{CAP}$ _01_01). A segunda coluna apresenta as funções da TRANSITIVIDADE do texto, por meio da sua distância topológica relativamente às outras funções (por exemplo: 14 = Processo: Material Transitivo e Transformativo / $39=$ Processo: Relacional Identificativo e Intensivo). A terceira coluna apresenta o MODO (por exemplo: $20=$ Modo: Declarativo). A quarta apresenta o TEMA (por exemplo: $10=$ Tema: Default $/ 45=$ Tema: Intensivo). A quinta coluna apresenta cada onda de informação. Esse tipo de quadro revela todas as orações do texto com a disposição da TRANSITIVIDADE, do MODO, do TEMA e das ondas de informação, permitindo, assim, a visualização da mudança entre orações.

${ }^{3}$ As tabelas e quadros desta pesquisa são de nossa autoria. 
Consequentemente, as mudanças de significado no manual podem ser visualizadas.

Além da visualização da mudança de significado do manual, para perfilação sistêmica, é necessário o cálculo das frequências relativas de cada categoria supracitada em relação ao corpus e às ondas de informação. O resultado desse cálculo aponta as probabilidades das escolhas dos sistemas gramaticais para o corpus combinado de manuais em inglês e em português brasileiro.

\section{Resultados da análise e discussão da construção de significado do corpus combinado}

Os quatro textos do corpus de manuais foram analisados pelo viés textual por meio das frequências obtidas do TEMA, interpessoal pelas frequências obtidas do MODO, ideacional pelas frequências obtidas da TRANSITIVIDADE e, por fim, pelas frequências das ondas de informação.

As frequências obtidas no TEMA explicam como a relação entre os significados foi construída. As frequências obtidas no MODO mostram a relação entre autor / leitor no discurso dos manuais. As frequências obtidas na TRANSITIVIDADE expõem como a representação das experiências do mundo se apresenta nesse tipo de texto. E a análise das ondas de informação aponta como a relação entre essas três categorias constroem o significado semântico de cada texto.

\subsection{Frequências relativas ao TEMA, MODO e TRANSITIVIDADE do manual Macbookpro Retina 13 inch em IO}

As Tabelas 1, 2 e 3 a seguir apresentam, respectivamente, as frequências relativas ao TEMA, MODO e TRANSITIVIDADE obtidas na análise do Manual Macbookpro Retina 13 inch em IO. As análises das categorias foram realizadas de forma semiautomática com o software UAM CorpusTool $^{\mathbb{O}}$ (O’DONNELL, 2008). 
Tabela 1 - Resultados da análise textual do Manual Macbookpro Retina 13 inch em IO

\begin{tabular}{l|c|c}
\hline \multicolumn{3}{c}{ CAP_01 } \\
\hline \multicolumn{1}{c}{ TEMA } & Frequência & $\mathrm{N}^{\mathrm{o}}$ de mensagens \\
\hline default & $84.3 \%$ & 75 \\
tema elemental & $0.0 \%$ & 0 \\
ângulo fonte & $0.0 \%$ & 0 \\
ângulo ponto de vista & $0.0 \%$ & 0 \\
tema perspectiva & $15.7 \%$ & 14 \\
tema intensivo & $0.0 \%$ & 0 \\
TOTAL & $100 \%$ & 87 \\
\hline
\end{tabular}

Tabela 2 - Resultados da análise Interpessoal do Manual Macbookpro Retina 13 inch em IO

\begin{tabular}{lcc}
\hline & CAP_01 & \\
\hline \multicolumn{1}{c}{ MODO } & Frequência & $\mathbf{N}^{\mathbf{0}} \mathbf{d e}$ \\
jussivo & $44.9 \%$ & 40 \\
sugestivo & $2.2 \%$ & 2 \\
declarativo & $52.8 \%$ & 47 \\
polar & $0.0 \%$ & 0 \\
elemental & $0.0 \%$ & 0 \\
\multicolumn{1}{r}{ TOTAL } & $\mathbf{1 0 0 \%}$ & $\mathbf{8 9}$ \\
\hline
\end{tabular}

Tabela 3 - Resultados da análise Ideacional do Manual Macbookpro Retina 13 inch em IO

(Continua)

\begin{tabular}{lrccc}
\hline \multicolumn{5}{c}{ CAP_01 } \\
\hline TIPO-DE-PROCESSO & & Frequência & $\mathbf{N}^{\mathbf{0}}$ de mensagens \\
\hline \multirow{3}{*}{ TIPO-DE-FAZER } & material & $74.2 \%$ & & $\mathbf{6 6}$ \\
& & & $\mathrm{N}=66$ & \\
& transformativo & $87.9 \%$ & & 58 \\
TIPO-DE-IMPACTO & criativo & $12.1 \%$ & & 8 \\
& & & $\mathrm{~N}=66$ & \\
& intransitivo & $7.6 \%$ & & 5 \\
& transitivo & $92.4 \%$ & & 61 \\
TIPO-DE-MENTAL & mental & $9.0 \%$ & & $\mathbf{8}$ \\
& & & $\mathrm{N}=8$ & \\
& superior & $87.5 \%$ & & 7 \\
& inferior & $12.5 \%$ & & 1 \\
\hline
\end{tabular}


Tabela 3 - Resultados da análise Ideacional do Manual Macbookpro Retina 13 inch em IO

(Conclusão)

\begin{tabular}{|c|c|c|}
\hline \multicolumn{3}{|c|}{ CAP_01 } \\
\hline TIPO-DE-PROCESSO & Frequência & $N^{0}$ de mensagens \\
\hline TIPO-DE-SUPERIOR & & $\mathrm{N}=7$ \\
\hline cognitivo & $100.0 \%$ & 7 \\
\hline desiderativo & $0.0 \%$ & 0 \\
\hline TIPO-DE-INFERIOR & & $\mathrm{N}=1$ \\
\hline perceptivo & $100.0 \%$ & 1 \\
\hline emotivo & $0.0 \%$ & 0 \\
\hline TIPO-DE-FENOMENALIZAÇÃO & & $\mathrm{N}=8$ \\
\hline fenomenalização & $75.0 \%$ & 6 \\
\hline não-fenomenalização & $25.0 \%$ & 2 \\
\hline verbal & $0.0 \%$ & $\mathbf{0}$ \\
\hline TIPO-DE-RECEPÇÃO & & $\mathrm{N}=0$ \\
\hline não-recepção & $0.0 \%$ & 0 \\
\hline recepção & $0.0 \%$ & 0 \\
\hline TIPO-DE-VERBAL & & $\mathrm{N}=0$ \\
\hline atividade & $0.0 \%$ & 0 \\
\hline semiose & $0.0 \%$ & 0 \\
\hline relacional & $13.5 \%$ & 12 \\
\hline TIPO-DE-RELAÇÃO & & $\mathrm{N}=12$ \\
\hline intensivo & $33.3 \%$ & 4 \\
\hline posessivo & $33.3 \%$ & 4 \\
\hline circunstancial & $33.3 \%$ & 4 \\
\hline MODO-DE-RELAÇÃO & & $\mathrm{N}=12$ \\
\hline atributivo & $58.3 \%$ & 7 \\
\hline identificativo & $41.7 \%$ & 5 \\
\hline existencial & $3.4 \%$ & 3 \\
\hline TIPO-DE-EXISTENCIAL & & $\mathrm{N}=3$ \\
\hline criação & $0.0 \%$ & 0 \\
\hline permanência & $100.0 \%$ & 3 \\
\hline TIPO-DE-CRIAÇÃO & & $\mathrm{N}=0$ \\
\hline surgimento & $0.0 \%$ & 0 \\
\hline extinção & $0.0 \%$ & 0 \\
\hline TIPO-PERMANÊNCIA & & $\mathrm{N}=3$ \\
\hline positiva & $100.0 \%$ & 3 \\
\hline negativa & $0.0 \%$ & 0 \\
\hline EXISTÊNCIA & & $\mathrm{N}=3$ \\
\hline introdução & $33.3 \%$ & 1 \\
\hline representação & $66.7 \%$ & 2 \\
\hline TOTAL & $100 \%$ & 89 \\
\hline
\end{tabular}


4.2 Análise das ondas de informação do manual Macbookpro Retina 13 inch em IO

O manual em IO é composto por quinze ondas de informação que juntas exercem a função de fazer com que o falante compreenda e utilize os aplicativos do Macbookpro Retina 13 inch. Elas são responsáveis por apresentar o manual, fazer explicações sobre os aplicativos do aparelho, explicar como conseguir mais informações sobre o notebook e dar informações sobre a garantia do produto.

Para construir esses significados, esse manual utiliza as funções: (1) EXPLICAR, ${ }^{4}$ (2) COMANDAR, (3) CLASSIFICAR, CONVIDAR e (5) INTRODUZIR.

(1) A função EXPLICAR é realizada pelo Processo: Material (14 $=$ Transitivo e Transformativo / $16-$ Transitivo e Criativo / $11-$ Intransitivo e Transformativo) com o Modo: Declarativo (20) e com o Tema: Default (10) ou Tema Perspectiva (41). Ela também pode ser realizada pelo Processo: Mental (56 - Perceptivo e Fenomenalização / 59 = Cognitivo e Fenomenalização) com o Modo: Declarativo (20) e com o Tema: Default (10) ou Tema Perspectiva (41).

A função (2) COMANDAR é realizada pelo Modo: Jussivo (11). Ele pode realizar com o Processo: Material (14/16/11) cosselecionando com o Tema: Default ou Tema: Perspectiva, ou com o Processo: Mental (59 - Cognitivo e Fenomenalização / 54 - Cognitivo e Não Fenomenalização) e com o Tema: Default (10).

A função (3) CLASSIFICAR ocorre no manual para caracterizar participantes e para auxiliar nas explicações, pois só por meio delas que o falante terá condições de compreender o que está sendo explicado. Estas informações novas são realizadas por Processo: Relacional (39= Processo: Relacional Identificativo e Intensivo / 37 - Identificativo e Circunstancial / 33 - Atributivo e Intensivo / 32 - Atributivo e Possessivo) que vem sempre acompanhado do Modo: Declarativo (20) e com o Tema: Default (10) ou Tema: Perspectiva (41).

4

As funções semânticas estão em caixa alta somente para melhorar a visualização do leitor. Elas não estão aqui representando sistemas como Martin (2013) propõe. 
A função (4) CONVIDAR é responsável por estimular o leitor a aprender sobre o notebook, causando, assim, proximidade. Para isso, o Modo: Sugestivo (15) foi utilizado sempre acompanhado do Processo: Material (14/11) e do Tema: Default (10).

A função (5) INTRODUZIR insere participantes novos e auxilia nas explicações; para isso, o Processo: Existencial: Permanência (29) é utilizado. No caso do IO, ele sempre se apresenta com o Modo: Declarativo (20) e com o Tema: Default (10) ou Tema: Perspectiva (41).

\subsection{Frequências relativas ao TEMA, MODO e TRANSITIVIDADE do manual Macbookpro Retina 13 inch em PT}

As Tabelas 4, 5 e 6 a seguir expõem, respectivamente, as frequências relativas ao TEMA, MODO e TRANSITIVIDADE obtidas na análise do Manual Macbookpro Retina 13 inch em PT:

Tabela 4 - Resultados da análise textual do Manual Macbookpro Retina 13 inch em PT

\begin{tabular}{lccc}
\hline \multicolumn{3}{c}{ CAP_02 } \\
\hline \multicolumn{1}{c}{ TEMA } & Frequência & $\mathbf{N}^{\mathbf{0}}$ de mensagens \\
\hline Default & $82.9 \%$ & 68 \\
tema elemental & $0.0 \%$ & 0 \\
ângulo fonte & $0.0 \%$ & 0 \\
ângulo ponto de vista & $0.0 \%$ & 0 \\
tema perspectiva & $0.0 \%$ & 0 \\
tema intensivo & $17.1 \%$ & 14 \\
TOTAL & $100 \%$ & 82 \\
\hline
\end{tabular}

Tabela 5 - Resultados da análise Interpessoal do Manual Macbookpro Retina 13 inch em PT

\begin{tabular}{lcc}
\hline \multicolumn{3}{c}{ CAP_02 } \\
\hline MODO & Frequência & $\mathbf{N}^{\mathbf{0}}$ de mensagens \\
\hline jussivo & $48.8 \%$ & 40 \\
sugestivo & $2.4 \%$ & 2 \\
declarativo & $48.8 \%$ & 40 \\
polar & $0.0 \%$ & 0 \\
elemental & $0.0 \%$ & 0 \\
TOTAL & $100 \%$ & 82 \\
\hline
\end{tabular}


Tabela 6 - Resultados da análise Ideacional do Manual Macbookpro Retina 13 inch em PT

(Continua)

\begin{tabular}{|c|c|c|c|}
\hline \multicolumn{4}{|c|}{ CAP_02 } \\
\hline TIPO-DE-PROCESSO & Frequência & $N^{0}$ de mensagens & \\
\hline material & $75.6 \%$ & & 62 \\
\hline TIPO-DE-FAZER & & $\mathrm{N}=62$ & \\
\hline transformativo & $90.3 \%$ & & 56 \\
\hline criativo & $9.7 \%$ & & 6 \\
\hline TIPO-DE-IMPACTO & & $\mathrm{N}=62$ & \\
\hline intransitivo & $1.6 \%$ & & 1 \\
\hline transitivo & $98.4 \%$ & & 61 \\
\hline mental & $8.5 \%$ & & 7 \\
\hline TIPO-DE-MENTAL & & $\mathrm{N}=7$ & \\
\hline superior & $85.7 \%$ & & 6 \\
\hline inferior & $14.3 \%$ & & 1 \\
\hline TIPO-DE-SUPERIOR & & $\mathrm{N}=6$ & \\
\hline cognitivo & $100.0 \%$ & & 5 \\
\hline desiderativo & $0.0 \%$ & & 0 \\
\hline TIPO-DE-INFERIOR & & $\mathrm{N}=1$ & \\
\hline perceptivo & $100.0 \%$ & & 1 \\
\hline emotivo & $0.0 \%$ & & 0 \\
\hline TIPO-DE-FENOMENALIZAÇÃO & & $\mathrm{N}=7$ & \\
\hline fenomenalização & $71.4 \%$ & & 5 \\
\hline não fenomenalização & $28.6 \%$ & & 2 \\
\hline verbal & $0.0 \%$ & & $\mathbf{0}$ \\
\hline TIPO-DE-RECEPÇÃO & & $\mathrm{N}=0$ & \\
\hline não-recepção & $0.0 \%$ & & 0 \\
\hline recepção & $0.0 \%$ & & 0 \\
\hline TIPO-DE-VERBAL & & $\mathrm{N}=0$ & \\
\hline atividade & $0.0 \%$ & & 0 \\
\hline semiose & $0.0 \%$ & & 0 \\
\hline relacional & $13.4 \%$ & & 11 \\
\hline TIPO-DE-RELAÇÃO & & $\mathrm{N}=11$ & \\
\hline intensivo & $27.3 \%$ & & 3 \\
\hline posessivo & $36.4 \%$ & & 4 \\
\hline circunstancial & $36.4 \%$ & & 4 \\
\hline MODO-DE-RELAÇÃO & & $\mathrm{N}=11$ & \\
\hline atributivo & $63.6 \%$ & & 7 \\
\hline identificativo & $36.4 \%$ & & 4 \\
\hline existencial & $2.4 \%$ & & 2 \\
\hline
\end{tabular}


Tabela 6 - Resultados da análise Ideacional do Manual Macbookpro Retina 13 inch em PT

(Conclusão)

\begin{tabular}{|c|c|c|c|}
\hline \multicolumn{4}{|c|}{ CAP 02} \\
\hline TIPO-DE-PROCESSO & Frequência & $\mathrm{N}^{0}$ de mensagens & \\
\hline TIPO-DE-EXISTENCIAL & & $\mathrm{N}=2$ & \\
\hline criação & $0.0 \%$ & & 0 \\
\hline permanência & $100.0 \%$ & & 3 \\
\hline TIPO-DE-CRIAÇÃO & & $\mathrm{N}=0$ & \\
\hline surgimento & $0.0 \%$ & & 0 \\
\hline extinção & $0.0 \%$ & & 0 \\
\hline TIPO-PERMANÊNCIA & & $\mathrm{N}=2$ & \\
\hline positiva & $100.0 \%$ & & 3 \\
\hline negativa & $0.0 \%$ & & 0 \\
\hline EXISTÊNCIA & & $\mathrm{N}=2$ & \\
\hline introdução & $50.0 \%$ & & 1 \\
\hline representação & $50.0 \%$ & & 2 \\
\hline TOTAL & $100 \%$ & 79 & \\
\hline
\end{tabular}

4.4 Análise das ondas de informação do manual Macbookpro Retina 13 inch em PT

O significado gerado pela junção de TRANSITIVIDADE, MODO e TEMA das mensagens e o significado macro gerado pela junção das mensagens compõem as ondas de informação (MARTIN; ROSE, 2007). Elas são responsáveis por apresentar o manual, fazer explicações sobre os aplicativos do aparelho, explicar como conseguir mais informações sobre ele e dar informações sobre a garantia do produto.

Para construir esses significados, esse manual utiliza as funções: (1) EXPLICAR, (2) COMANDAR, (3) CLASSIFICAR, CONVIDAR e (5) INTRODUZIR.

A função (1) EXPLICAR é realizada pelo Processo: Material (14 $=$ Transitivo e Transformativo / $16-$ Transitivo e Criativo) com o Modo: Declarativo (20) e com o Tema: Default (10) ou Tema Perspectiva (41). Ela também pode ser realizada pelo Processo: Mental $(59=$ Cognitivo e Fenomenalização) com o Modo: Declarativo (20) e com o Tema Perspectiva (41). 
A função (2) COMANDAR é realizada pelo Modo: Jussivo (11). Ele pode realizar com o Processo: Material (14/16) cosselecionando com o Tema: Default ou Tema: Perspectiva, ou com o Processo: Mental (56 Perceptivo e Fenomenalização / 59 - Cognitivo e Fenomenalização / 54 Cognitivo e Não Fenomenalização) e com o Tema: Default (10).

A função (3) CLASSIFICAR ocorre no manual para caracterizar participantes e para auxiliar nas explicações, pois só por meio delas o falante terá condições de compreender o que está sendo explicado. Essas informações novas são realizadas por Processo: Relacional (33 Atributivo e Intensivo / 32 - Atributivo e Possessivo / 37 - Identificativo e Circunstancial) que vem sempre acompanhado do Modo: Declarativo (20) e com o Tema: Default (10) ou Tema: Perspectiva (41).

A função (4) CONVIDAR é responsável por estimular o leitor a aprender sobre o notebook, causando, assim, uma proximidade. Para isso, o Modo: Sugestivo foi utilizado sempre acompanhado do Processo: Material (14/11) e do Tema: Default (10).

A função (5) INTRODUZIR insere participantes novos e auxilia nas explicações, para isso, o Processo: Existencial: Permanência (29) é utilizado. No caso do PT, ele se apresenta com o Modo: Declarativo (20) e com o Tema: Default (10) ou Tema: Perspectiva (41).

4.5 Frequências relativas ao TEMA, MODO e TRANSITIVIDADE do manual Panela de Pressão Solar em PO

As Tabelas 7, 8 e 9 a seguir mostram, respectivamente, as frequências relativas ao TEMA, MODO e TRANSITIVIDADE obtidas na análise do Manual Panela de Pressão Solar em PO. 
Tabela 7 - Resultados da análise textual do Manual Panela de Pressão Solar em PO

\begin{tabular}{lccc}
\hline \multicolumn{3}{c}{ CAP_03 } \\
\hline \multicolumn{1}{c}{ TEMA } & Frequência & $\mathbf{N}^{\mathbf{0}}$ de mensagens \\
\hline default & $61.9 \%$ & 39 \\
tema elemental & $0.0 \%$ & 0 \\
ângulo fonte & $0.0 \%$ & 0 \\
anngulo ponto de vista & $0.0 \%$ & 0 \\
tema perspectiva & $38.1 \%$ & 24 \\
tema intensivo & $0.0 \%$ & 0 \\
& TOTAL & $100 \%$ & 63 \\
\hline
\end{tabular}

Tabela 8 - Resultados da análise Interpessoal do Manual Panela de Pressão Solar em PO

\begin{tabular}{lccc}
\hline \multicolumn{3}{c}{ CAP_03 } \\
\hline \multicolumn{1}{c}{ MODO } & Frequência & $\mathbf{N}^{\mathbf{0}}$ de mensagens \\
\hline jussivo & $55.6 \%$ & 35 \\
sugestivo & $0.0 \%$ & 0 \\
declarativo & $44,4 \%$ & 28 \\
polar & $0.0 \%$ & 0 \\
elemental & $0.0 \%$ & 0 \\
\multicolumn{1}{c}{ TOTAL } & $\mathbf{1 0 0 \%}$ & $\mathbf{6 3}$ \\
\hline
\end{tabular}

Tabela 9 - Resultados da análise Ideacional do Manual Panela de Pressão Solar em PO

(Continua)

\begin{tabular}{|c|c|c|}
\hline \multicolumn{3}{|c|}{ CAP_03 } \\
\hline TIPO-DE-PROCESSO & Frequência & $\begin{array}{l}\mathbf{N}^{0} \text { de } \\
\text { mensagens }\end{array}$ \\
\hline TIPO-DE-FAZER & $\begin{array}{l}77.8 \% \\
\quad \mathrm{~N}=49\end{array}$ & 49 \\
\hline transformativo & $95.9 \%$ & 47 \\
\hline TIPO-DE-IMPACTO $\quad$ criativo & $\begin{array}{l}4.1 \% \\
\mathrm{~N}=49\end{array}$ & 2 \\
\hline intransitivo & $0.0 \%$ & 0 \\
\hline transitivo & $100.0 \%$ & 49 \\
\hline mental & $9.5 \%$ & 6 \\
\hline
\end{tabular}


Tabela 9 - Resultados da análise Ideacional do Manual Panela de Pressão Solar em PO

(Conclusão)

\begin{tabular}{|c|c|c|c|}
\hline \multicolumn{4}{|c|}{ CAP_03 } \\
\hline TIPO-DE-PROCESSO & Frequência & \multicolumn{2}{|c|}{$\begin{array}{l}\mathbf{N}^{0} \text { de } \\
\text { mensagens }\end{array}$} \\
\hline TIPO-DE-MENTAL & \multicolumn{3}{|c|}{$\mathrm{N}=6$} \\
\hline superior & $100.0 \%$ & & 6 \\
\hline inferior & $0.0 \%$ & & 0 \\
\hline TIPO-DE-SUPERIOR & \multicolumn{3}{|c|}{$\mathrm{N}=6$} \\
\hline cognitivo & $100.0 \%$ & & 6 \\
\hline desiderativo & $0.0 \%$ & & 0 \\
\hline TIPO-DE-INFERIOR & \multicolumn{3}{|c|}{$\mathrm{N}=0$} \\
\hline perceptivo & $0.0 \%$ & & 0 \\
\hline emotivo & \multirow{2}{*}{\multicolumn{3}{|c|}{$\begin{array}{l}0.0 \% \\
\quad \mathrm{~N}=6\end{array}$}} \\
\hline TIPO-DE-FENOMENALIZAÇÃO & & & \\
\hline fenomenalização & $100.0 \%$ & & 6 \\
\hline não fenomenalização & $0.0 \%$ & & 0 \\
\hline verbal & $3.2 \%$ & & 2 \\
\hline TIPO-DE-RECEPÇÃO & \multicolumn{3}{|c|}{$\mathrm{N}=2$} \\
\hline não-recepção & $100.0 \%$ & & 2 \\
\hline recepção & $0.0 \%$ & & 0 \\
\hline TIPO-DE-VERBAL & \multicolumn{3}{|c|}{$\mathrm{N}=2$} \\
\hline atividade & $0.0 \%$ & & 0 \\
\hline semiose & $100.0 \%$ & & 2 \\
\hline relacional & \multirow{2}{*}{\multicolumn{3}{|c|}{$\begin{array}{l}7.9 \% \\
\quad \mathrm{~N}=5\end{array}$}} \\
\hline TIPO-DE-RELAÇÃO & & & \\
\hline intensivo & $60.0 \%$ & & 3 \\
\hline posessivo & $40.0 \%$ & & 4 \\
\hline circunstancial & $0.0 \%$ & & 0 \\
\hline MODO-DE-RELAÇÃO & \multicolumn{3}{|c|}{$\mathrm{N}=5$} \\
\hline atributivo & $100.0 \%$ & & 4 \\
\hline identificativo & $0.0 \%$ & & 0 \\
\hline existencial & $1.6 \%$ & & 1 \\
\hline TIPO-DE-EXISTENCIAL & \multicolumn{3}{|c|}{$\mathrm{N}=1$} \\
\hline criação & $0.0 \%$ & & 0 \\
\hline permanência & $100.0 \%$ & & 1 \\
\hline TIPO-DE-CRIAÇÃO & \multicolumn{3}{|c|}{$\mathrm{N}=0$} \\
\hline surgimento & $0.0 \%$ & & 0 \\
\hline extinção & $0.0 \%$ & & 0 \\
\hline TIPO-PERMANÊNCIA & \multicolumn{3}{|c|}{$\mathrm{N}=1$} \\
\hline positiva & $100.0 \%$ & & 1 \\
\hline negativa & \multirow{2}{*}{\multicolumn{3}{|c|}{$\mathrm{N}=1$}} \\
\hline EXISTÊNCIA & & & \\
\hline introdução & $100.0 \%$ & & 1 \\
\hline representação & $0.0 \%$ & & 0 \\
\hline TOTAL & $100 \%$ & 63 & \\
\hline
\end{tabular}


4.6 Análise das ondas de informação do manual Panela de Pressão Solar em PO

O manual em PO é realizado por 18 ondas de informação que, juntas, exercem a função de habilitar o leitor a compreender a forma correta de manusear a panela de pressão, evitando, assim, acidentes domésticos. As ondas de informação são responsáveis por apresentar o manual, advertir o leitor, dar comandos para que o leitor use corretamente a panela, fazer explicações para informar porque o leitor deve obedecer aos comandos e explicar questões envolvidas no uso e na manutenção da panela.

Para construir esses significados, esse manual utiliza as funções:
(1) EXPLICAR
(2) COMANDAR,
(3)
CLASSIFICAR e INTRODUZIR.

A função (1) EXPLICAR é realizada pelo Processo: Material (14 $=$ Transitivo e Transformativo $/ 16-$ Transitivo e Criativo) com o Modo: Declarativo (20) e com o Tema: Default (10) ou Tema Perspectiva (41). Ela também pode ser realizada pelo Processo: Mental $(59=$ Cognitivo e Fenomenalização) com o Modo: Declarativo (20) e com o Tema Perspectiva (41).

A função (2) COMANDAR é realizada pelo Modo: Jussivo (11). Ele pode realizar com o Processo: Material (14/16) cosselecionando com o Tema: Default ou Tema: Perspectiva ou com o Processo: Mental (59 Cognitivo e Fenomenalização) e com o Tema: Default (10) ou Tema Perspectiva (41).

A função (3) CLASSIFICAR ocorre no manual para caracterizar participantes e para auxiliar nas explicações. Essas informações novas são realizadas por Processo: Relacional (33 - Atributivo e Intensivo / 32 - Atributivo e Possessivo) que vem sempre acompanhado do Modo: Declarativo (20) e com o Tema: Default (10) ou Tema: Perspectiva (41).

A função (4) INTRODUZIR insere participantes novos e significados novos no texto e auxilia nas explicações, para isso, o Processo: Existencial: Permanência (29) é utilizado e o Processo: Verbal (41 - Semiose e Não Recepção). No caso do Processo: Existencial, ele vem com o Modo: Declarativo (20) e com o Tema: Default (10) e o 
Processo: Verbal vem acompanhado do Modo: Declarativo (20) e com o Tema: Default (10) ou o Tema: Perspectiva.

4.7 Frequências relativas ao TEMA, MODO e TRANSITIVIDADE do manual Panela de Pressão Solar em IT

As Tabelas 10, 11 e 12 a seguir apresentam, respectivamente, as frequências relativas ao TEMA, MODO e TRANSITIVIDADE obtidas na análise do Manual Panela de Pressão Solar em IT.

Tabela 10 - Resultados da análise textual do Manual Panela de Pressão Solar em IT

\begin{tabular}{l|c|c}
\hline \multicolumn{3}{c}{ CAP 04 } \\
\hline \multicolumn{1}{c}{ TEMA } & Frequência & $\mathbf{N}^{\mathbf{0}}$ de mensagens \\
\hline default & $54.0 \%$ & 34 \\
tema elemental & $0.0 \%$ & 0 \\
angulo fonte & $0.0 \%$ & 0 \\
ângulo ponto de vista & $0.0 \%$ & 0 \\
tema perspectiva & $46.0 \%$ & 29 \\
tema intensivo & $0.0 \%$ & 0 \\
\multicolumn{1}{c}{ TOTAL } & $\mathbf{1 0 0 \%}$ & $\mathbf{6 3}$ \\
\hline
\end{tabular}

Tabela 11 - Resultados da análise Interpessoal do Manual Panela de Pressão Solar em IT

\begin{tabular}{|c|c|c|}
\hline \multicolumn{3}{|c|}{ CAP 04} \\
\hline MODO & Frequência & $\mathrm{N}^{0}$ de mensagens \\
\hline jussivo & $61.9 \%$ & 39 \\
\hline sugestivo & $0.0 \%$ & 0 \\
\hline declarativo & $38.1 \%$ & 24 \\
\hline polar & $0.0 \%$ & 0 \\
\hline elemental & $0.0 \%$ & 0 \\
\hline TOTAL & $100 \%$ & 63 \\
\hline
\end{tabular}


Tabela 12 - Resultados da análise Ideacional do Manual Panela de Pressão Solar em IT

(Continua)

\begin{tabular}{|c|c|c|c|}
\hline \multicolumn{4}{|c|}{ CAP_04 } \\
\hline TIPO-DE-PROCESSO & Frequência & $\mathrm{N}^{0} \mathrm{~d}$ & \\
\hline material & $77.8 \%$ & & 49 \\
\hline TIPO-DE-FAZER & & $\mathrm{N}=49$ & \\
\hline transformativo & $98.0 \%$ & & 48 \\
\hline criativo & $2.0 \%$ & & 1 \\
\hline TIPO-DE-IMPACTO & & $\mathrm{N}=49$ & \\
\hline intransitivo & $0.0 \%$ & & 0 \\
\hline transitivo & $100.0 \%$ & & 49 \\
\hline mental & $4.8 \%$ & & 3 \\
\hline TIPO-DE-MENTAL & & $\mathrm{N}=3$ & \\
\hline superior & $100.0 \%$ & & 3 \\
\hline inferior & $0.0 \%$ & & 0 \\
\hline TIPO-DE-SUPERIOR & & $\mathrm{N}=3$ & \\
\hline cognitivo & $100.0 \%$ & & 3 \\
\hline desiderativo & $0.0 \%$ & & 0 \\
\hline TIPO-DE-INFERIOR & & $\mathrm{N}=0$ & \\
\hline perceptivo & $0.0 \%$ & & 0 \\
\hline emotivo & $0.0 \%$ & & 0 \\
\hline TIPO-DE-FENOMENALIZAÇÃO & & $\mathrm{N}=3$ & \\
\hline fenomenalização & $100.0 \%$ & & 3 \\
\hline não fenomenalização & $0.0 \%$ & & 0 \\
\hline verbal & $3.2 \%$ & & 2 \\
\hline TIPO-DE-RECEPÇÃO & & $\mathrm{N}=2$ & \\
\hline não-recepção & $100.0 \%$ & & 2 \\
\hline recepção & $0.0 \%$ & & 0 \\
\hline TIPO-DE-VERBAL & & $\mathrm{N}=2$ & \\
\hline atividade & $0.0 \%$ & & 0 \\
\hline semiose & $100.0 \%$ & & 2 \\
\hline relacional & $12.7 \%$ & & 8 \\
\hline TIPO-DE-RELAÇÃO & & $\mathrm{N}=8$ & \\
\hline intensivo & $87.5 \%$ & & 7 \\
\hline posessivo & $12.5 \%$ & & 1 \\
\hline circunstancial & $0.0 \%$ & & 0 \\
\hline MODO-DE-RELAÇÃO & & $\mathrm{N}=8$ & \\
\hline atributivo & $87.5 \%$ & & 7 \\
\hline identificativo & $12.5 \%$ & & 1 \\
\hline existencial & $1.6 \%$ & & 1 \\
\hline
\end{tabular}


Tabela 12 - Resultados da análise Ideacional do Manual Panela de Pressão Solar em IT

(Conclusão)

\begin{tabular}{|c|c|c|c|}
\hline \multicolumn{4}{|c|}{ CAP_04 } \\
\hline TIPO-DE-PROCESSO & Frequência & $N^{0}$ de mensagens & \\
\hline TIPO-DE-EXISTENCIAL & & $\mathrm{N}=1$ & \\
\hline criação & $0.0 \%$ & & 0 \\
\hline permanência & $100.0 \%$ & & 1 \\
\hline TIPO-DE-CRIAÇÃO & & $\mathrm{N}=0$ & \\
\hline surgimento & $0.0 \%$ & & 0 \\
\hline extinção & $0.0 \%$ & & 0 \\
\hline TIPO-PERMANÊNCIA & & $\mathrm{N}=1$ & \\
\hline positiva & $100.0 \%$ & & 1 \\
\hline negativa & $0.0 \%$ & & 0 \\
\hline EXISTÊNCIAL & & $\mathrm{N}=1$ & \\
\hline introdução & $100.0 \%$ & & 1 \\
\hline representação & $0.0 \%$ & & 0 \\
\hline TOTAL & $100 \%$ & & 63 \\
\hline
\end{tabular}

4.8 Análise das ondas de informação do manual Panela de Pressão Solar em IT

O manual em IT é composto por 18 ondas de informação que auxiliam o leitor a compreender a forma correta de usar a panela de pressão Solar da Tramontina. As ondas de informação são responsáveis por apresentar o manual, advertir o leitor, dar comandos para que o leitor use corretamente a panela, dar explicações para informar por que o leitor deve obedecer aos comandos e explicar questões envolvidas no uso e na manutenção da panela.

Para construir esses significados, esse manual utiliza as funções: (1) EXPLICAR, (2) COMANDAR, (3) CLASSIFICAR e (4) INTRODUZIR.

A função EXPLICAR (1) é realizada pelo Processo: Material (14 $=$ Transitivo e Transformativo / 16 - Transitivo e Criativo) com o Modo: Declarativo (20) e com o Tema: Default (10) ou Tema Perspectiva (41). Ela também pode ser realizada pelo Processo: Mental $(59=$ Cognitivo e Fenomenalização) com o Modo: Declarativo (20) e com o Tema Perspectiva (41). 
A função (2) COMANDAR é realizada pelo Modo: Jussivo (11). Ele pode realizar com o Processo: Material (14) cosselecionando com o Tema: Default ou Tema: Perspectiva, ou com o Processo: Mental (59Cognitivo e Fenomenalização) e com o Tema: Perspectiva (41), ou com o Processo Relacional (33 - Intensivo e Atributivo) cosselecionando com o Tema: Default (10) ou Tema: Perspectiva (41).

A função (3) CLASSIFICAR ocorre no manual para caracterizar participantes e para auxiliar nas explicações. Estas informações novas são realizadas por Processo: Relacional (39 - Processo: Relacional Identificativo e Intensivo 33 - Atributivo e Intensivo / 32 - Atributivo e Possessivo) que vem sempre acompanhado do Modo: Declarativo (20) e com o Tema: Default (10) ou Tema: Perspectiva (41).

A função (4) INTRODUZIR insere participantes novos e significados novos no texto e auxilia nas explicações, para isso, o Processo: Existencial: Permanência (29) é utilizado e o Processo: Verbal (41 - Semiose e Não Recepção). No caso do Processo: Existencial ele vem com o Modo: Declarativo (20) e com o Tema: Default (10) e o Processo: Verbal vem acompanhado do Modo: Declarativo (20) e com o Tema: Default (10).

\section{Comparação linguística do perfil sistêmico dos manuais}

Para a comparação linguística entre os manuais, Catford (1965, p. 27) foi utilizado quanto à comparação entre línguas e Krzeszowski (1990, p. 4) em relação à linguística contrastiva.

Ambos os autores apontam que as línguas podem ser comparadas à medida que elas possuam categorias em comum. Nesse sentido, para a análise do corpus desta pesquisa, foram usadas as categorias comuns da TRANSITIVIDADE, MODO e TEMA entre o inglês e português brasileiro. Sendo assim, os manuais são estruturalmente semelhantes e, portanto, comparáveis.

A seguir, apresentam-se as comparações entre os manuais. 


\subsection{Comparação OI e PT}

Comparando os textos pelas categorias gramaticais, é possível apontar equivalência textual e correspondência formal segundo Catford (1965, p. 27) entre o inglês e o português brasileiro.

Para esta análise, o Quadro 2 a seguir apresenta o perfil dos manuais em relação às categorias gramaticais utilizadas na análise e a função semântica gerada pela confluência de TRANSITIVIDADE, MODO e TEMA:

Quadro 2 - Comparação do perfil sistêmico entre os manuais IO e PT

\begin{tabular}{|c|c|c|c|c|c|c|c|}
\hline \multicolumn{4}{|c|}{$\begin{array}{l}\text { MANUAL INGLÊS } \\
\text { ORIGINAL }\end{array}$} & \multicolumn{4}{|c|}{$\begin{array}{l}\text { MANUAL PORTUGUÊS } \\
\text { TRADUZIDO }\end{array}$} \\
\hline \multicolumn{4}{|c|}{89 Mensagens / 15 Ondas de Informação } & \multicolumn{4}{|c|}{$\begin{array}{l}82 \text { Mensagens / } 14 \text { Ondas de } \\
\text { Informação }\end{array}$} \\
\hline \multicolumn{3}{|c|}{$\begin{array}{l}\text { Cosseleções } \\
\text { Gramaticais }\end{array}$} & \multirow[t]{2}{*}{$\begin{array}{l}\text { Funções } \\
\text { Semânticas }\end{array}$} & \multicolumn{3}{|c|}{$\begin{array}{l}\text { Cosseleções } \\
\text { Gramaticais }\end{array}$} & \multirow[t]{2}{*}{$\begin{array}{c}\text { Funções } \\
\text { Semânticas }\end{array}$} \\
\hline Trans. & Modo & Tema & & Trans. & Modo & Tema & \\
\hline $14 \backslash 16 \backslash 11$ & 20 & $10 \backslash 41$ & & $14 \backslash 16$ & 20 & $10 \backslash 41$ & \\
\hline $56 \backslash 59$ & 20 & $10 \backslash 41$ & EXPLICAR & 59 & 20 & 41 & EXPLICAR \\
\hline $14 \backslash 16 \backslash 11$ & 11 & $10 \backslash 41$ & & $14 \backslash 16$ & 11 & $10 \backslash 41$ & \\
\hline $59 \backslash 54$ & 11 & 10 & COMANDAR & $56 \backslash 59 \backslash 54$ & 11 & 10 & COMANDAR \\
\hline $39 \backslash 37 \backslash 33 \backslash 32$ & 20 & $10 \backslash 41$ & CLASSIFICAR & $37 \backslash 33 \backslash 32$ & 20 & $10 \backslash 41$ & CLASSIFICAR \\
\hline $14 \backslash 11$ & 15 & 10 & CONVIDAR & $14 \backslash 11$ & 15 & 10 & CONVIDAR \\
\hline 29 & 20 & $10 \backslash 41$ & INTRODUZIR & 29 & 20 & $10 \backslash 41$ & INTRODUZIR \\
\hline
\end{tabular}

Como mostra o Quadro 2, apesar de os manuais possuírem o mesmo número de tokens, o manual em IO traz 89 mensagens, ao passo que o manual em PT traz 82 mensagens. A mensagem 82 do manual em PT equivale à mensagem 80 do manual em IO. Mediante esse dado, pode-se afirmar que o manual em português precisa de mais tokens para 'significar' do que o manual em inglês e, consequentemente, mais mensagens.

Comparando pelo viés das ondas de informação, o manual em IO possui 15 ondas de informação e o manual em PT 14 ondas de informação. Pela função de cada uma, pode-se afirmar que existe equivalência textual e correspondência formal entre elas, pois a função semântica de uma onda na língua fonte foi traduzida para a mesma 
função semântica na língua alvo e, consequentemente, as categorias semânticas foram mantidas. Apesar de as funções "Como usar as pastas." e "Como usar o Launchpad" terem sido trocadas de ordem no texto alvo, a equivalência e a correspondência se mantiveram.

O quadro também aponta que as funções semânticas EXPLICAR, COMANDAR, CLASSIFICAR, CONVIDAR e INTRODUZIR são correspondentes na língua fonte e na língua alvo, todavia, a equivalência ocorre parcialmente, pois existem classes utilizadas para formar as funções semânticas em uma língua que não ocorrem na outra língua. Essas classes estão marcadas em negrito no Quadro 2 e estão especificadas a seguir.

O manual em inglês possui mais opções para a cosseleção do que o manual em PT. Para a função EXPLICAR, o manual em IO tem a opção de cosselecionar o Processo $11^{5}$ com o Modo 20 e o Tema 10/41; essa opção não existe no manual em PT. Para a mesma função, a opção de cosseleção com o Processo 56 com o Modo 20 e o Tema 10/41 não possui no PT, assim como a cosseleção do Processo 59 com o Modo 20 e o Tema 10.

Isso também ocorre na função COMANDAR, o manual em IO tem a opção de cosselecionar o Processo 11 com o Modo 20 e o Tema 10/41, opção não existente na tradução. No PT, a cosseleção com Processo 56 com o Modo 11 e o Tema 10 não ocorre no IO.

$\mathrm{Na}$ função CLASSIFICAR, existe a opção no manual em IO de cosselecionar o Processo 39 com o Modo 11 e o Tema 10/41, opção esta que não existe no manual traduzido.

As classes gramaticais da TRANSITIVIDADE, MODO e TEMA da língua fonte que foram mantidas na língua alvo apresentam a correspondência formal entre o inglês original e o português brasileiro traduzido no nível gramatical.

\footnotetext{
${ }^{5}$ Para saber a descrição gramatical do deslocamento no espaço gramatical, consultar análise das ondas de informação da seção anterior.
} 
Comparando com base nas classes gramaticais analisadas e nas cosseleções possíveis entre elas, é possível apontar equivalência textual e correspondência formal entre original e tradução.

O Quadro 3 a seguir apresenta o perfil dos manuais com as possíveis categorias gramaticais e as possíveis confluências entre elas, como também a realização semântica de cada uma:

Quadro 3 - Comparação do perfil sistêmico entre os manuais PO e IT

\begin{tabular}{|c|c|c|c|c|c|c|c|}
\hline \multirow{2}{*}{\multicolumn{4}{|c|}{$\begin{array}{c}\text { MANUAL PORTUGUÊS ORIGINAL } \\
63 \text { Mensagens / } 18 \text { Ondas de } \\
\text { Informação }\end{array}$}} & \multicolumn{4}{|c|}{ MANUAL INGLÊS TRADUZIDO } \\
\hline & & & & \multicolumn{4}{|c|}{$\begin{array}{l}63 \text { Mensagens / } 18 \text { Ondas de } \\
\text { Informação }\end{array}$} \\
\hline \multicolumn{3}{|c|}{$\begin{array}{l}\text { Cosseleções } \\
\text { Gramaticais }\end{array}$} & $\begin{array}{c}\text { Funções } \\
\text { Semânticas }\end{array}$ & \multicolumn{3}{|c|}{$\begin{array}{l}\text { Cosseleções } \\
\text { Gramaticais }\end{array}$} & \multirow[t]{2}{*}{$\begin{array}{l}\text { Funções } \\
\text { Semânticas }\end{array}$} \\
\hline Trans. & Modo & Tema & & Trans. & Modo & Tema & \\
\hline $14 \backslash 16$ & 20 & $10 \backslash 41$ & & $14 \backslash 16$ & 20 & $10 \backslash 41$ & \\
\hline 59 & 20 & 41 & EXPLICAR & 59 & 20 & 41 & EXPLICAR \\
\hline $14 \backslash 16$ & 11 & $10 \backslash 41$ & & 14 & 11 & $10 \backslash 41$ & \\
\hline 59 & 11 & $10 \backslash 41$ & COMANDAR & 59 & 11 & 41 & \\
\hline $33 \backslash 32$ & 20 & $10 \backslash 41$ & CLASSIFICAR & 33 & 11 & $10 \backslash 41$ & COMANDAR \\
\hline 29 & 20 & 10 & & $39 \backslash 33 \backslash 32$ & 20 & $10 \backslash 41$ & CLASSIFICAR \\
\hline \multirow[t]{2}{*}{41} & 20 & $10 \backslash 41$ & INTRODUZIR & 29 & 20 & 10 & \\
\hline & & & & 41 & 20 & 10 & INTRODUZIR \\
\hline
\end{tabular}

O Quadro 3 aponta que tanto o manual em PO quanto o manual em IT possuem 63 mensagens, porém a $63^{\mathrm{a}}$ mensagem do manual em PO equivale a $61^{\mathrm{a}}$ mensagem do manual em IT. Dessa forma, como na comparação da seção anterior, o manual em português brasileiro precisa de mais tokens e, por conseguinte, mais mensagens para significar do que o manual em inglês. Além da mesma quantidade de mensagens, os dois manuais possuem 18 ondas de informação.

Comparando as ondas de informação dos manuais, a análise realizada na seção anterior mostra que existe equivalência textual e correspondência formal entre elas, pois a função semântica de uma onda na língua fonte foi traduzida para a mesma função semântica na língua alvo e, consequentemente, as categorias semânticas foram mantidas. 
O Quadro 14 também apresenta as funções semânticas EXPLICAR, COMANDAR, CLASSIFICAR e INTRODUZIR do manual em PO que foram mantidas no IT, ocorrendo assim uma correspondência formal. No entanto, a equivalência ocorre parcialmente entre as funções semânticas, pois algumas classes gramaticais que foram utilizadas para formar a função semântica na língua fonte não foram utilizadas na língua alvo. Essas classes estão marcadas em negrito no Quadro 3 e estão especificadas a seguir.

Na função semântica COMANDAR do manual em PO, a opção de confluência entre o Processo 16 com o Modo 11 e o Tema 10/41 não existe no manual em IT. Também na mesma função semântica, a cosseleção entre o Tema 10, o Modo 11 e o Processo 59 no manual em PO não ocorre em IT.

Na função INTRODUZIR, o manual em PO possui a alternativa de confluência entre o Tema 41 com o Modo 20 e o Processo 41 que não ocorre no IT.

Comparando com base no no manual em IT, pode-se afirmar que ele, como na comparação da seção anterior, possui mais opções de cosseleção gramatical do que o manual em PO. Um exemplo disso ocorre na função COMANDAR do manual em IT, ele possui uma opção a mais de cosseleção do que o manual em PO. Esse manual em inglês usa o Processo 33 cosselecionando com o Modo 11 e o Tema 10/41 para dar ordens ao leitor.

$\mathrm{Na}$ função semântica CLASSIFICAR do mesmo manual, o Processo 39 cosseleciona com o Modo 20 e o Tema 10/4. Essa opção não existe no manual em PO.

As demais cosseleções gramaticais da língua fonte que foram mantidas na língua alvo apresentam a correspondência formal entre o português brasileiro original e o inglês traduzido no nível gramatical.

\subsection{Comparação IO e PO}

Pela análise realizada, os manuais em inglês original e em português original, podem ser comparadas em relação às categorias gramaticais utilizadas, as cosseleções entre os sistemas, as funções 
semânticas e as ondas de informação, a fim de apresentar a distância e a proximidade entre as línguas.

O Quadro 4 mostra o perfil dos manuais com as categorias gramaticais utilizadas e as confluências entre elas, como também a realização semântica de cada uma.

Quadro 4 - Comparação do perfil sistêmico entre os manuais IO e PO

\begin{tabular}{|c|c|c|c|c|c|c|c|}
\hline \multicolumn{4}{|c|}{$\begin{array}{l}\text { MANUAL INGLÊS } \\
\text { ORIGINAL }\end{array}$} & \multicolumn{4}{|c|}{$\begin{array}{c}\text { MANUAL PORTUGUES } \\
\text { ORIGINAL }\end{array}$} \\
\hline \multicolumn{4}{|c|}{$\begin{array}{l}89 \text { Mensagens / } 15 \text { Ondas de } \\
\text { Informação }\end{array}$} & \multicolumn{4}{|c|}{$\begin{array}{l}63 \text { Mensagens / } 18 \text { Ondas de } \\
\text { Informação }\end{array}$} \\
\hline \multicolumn{3}{|c|}{$\begin{array}{l}\text { Cosseleções } \\
\text { Gramaticais }\end{array}$} & \multirow[t]{2}{*}{$\begin{array}{c}\text { Funções } \\
\text { Semânticas }\end{array}$} & \multicolumn{3}{|c|}{$\begin{array}{l}\text { Cosseleções } \\
\text { Gramaticais }\end{array}$} & \multirow[t]{2}{*}{$\begin{array}{c}\text { Funções } \\
\text { Semânticas }\end{array}$} \\
\hline Trans. & Modo & Tema & & Trans. & Modo & Tema & \\
\hline $14 \backslash 16 \backslash \mathbf{1 1}$ & 20 & $10 \backslash 41$ & & $14 \backslash 16$ & 20 & $10 \backslash 41$ & \\
\hline $56 \backslash 59$ & 20 & $\mathbf{1 0} \backslash 41$ & EXPLICAR & 59 & 20 & 41 & EXPLICAR \\
\hline $14 \backslash 16 \backslash \mathbf{1 1}$ & 11 & $10 \backslash 41$ & & $14 \backslash 16$ & 11 & $10 \backslash 41$ & \\
\hline $59 \backslash 54$ & 11 & 10 & COMANDAR & 59 & 11 & $10 \backslash 41$ & COMANDAR \\
\hline $39 \backslash 37 \backslash 33 \backslash 32$ & 20 & $10 \backslash 41$ & CLASSIFICAR & $33 \backslash 32$ & 20 & $10 \backslash 41$ & CLASSIFICAR \\
\hline $14 \backslash 11$ & 15 & 10 & CONVIDAR & 29 & 20 & 10 & \\
\hline 29 & 20 & $10 \backslash 41$ & INTRODUZIR & 41 & 20 & $10 \backslash 41$ & INTRODUZIR \\
\hline
\end{tabular}

O Quadro 4 mostra que o manual em IO possui 89 mensagens, ao passo que o manual em PO possui 63 mensagens. Assim sendo, o manual do MacBook Pro precisa de mais mensagens para significar do que o manual da Panela de Pressão Solar.

Todavia, apesar de possuir mais mensagens, o manual em IO possui menos ondas de informação do que o manual em PO. Enquanto o primeiro apresenta 15 ondas; o segundo possui 18.

Apesar da quantidade de ondas de informação ser diferente e apesar dos manuais tratarem de objetos diferentes, eles apresentam duas funções para as ondas de informação que são semelhantes: a função "Apresentação", que é responsável por introduzir o manual, e a função "Primeiros passos para usar o MacBook Pro" e "Primeiros passos para usar a Panela de Pressão Solar”, que explica o que é necessário saber para começar a usar o computador ou a panela de pressão. 
O Quadro 4 aponta também as funções semânticas do manual em cada língua e suas respectivas cosseleções entre as classes gramaticais da TRANSITIVIDADE, MODO e TEMA.

Comparando os manuais a partir do manual em IO, a função CONVIDAR constituída a partir da confluência entre o Processo 14/11 com o Modo15 e o Tema 10 do manual em IO não ocorre no manual em PO.

$\mathrm{Na}$ função EXPLICAR, a confluência entre o Processo 11 com o Modo 20 e o Tema 10/41 não ocorre em português brasileiro. Também nessa mesma função, a confluência entre o Processo 56 com o Modo 20 e o Tema10/41 não ocorre no manual em PO. Com relação ao Tema 10, a confluência entre ele e o Modo20 e Processo 59 não existe em português brasileiro.

$\mathrm{Na}$ função COMANDAR, Processo 11 cosseleciona com o Modo 11 e com o Tema 10/41. Essa opção não existe em PO. Nessa mesma função semântica, o Processo 54 cosseleciona com o Modo 11 e com o Tema 10/41. Essa opção não existe em PO.

$\mathrm{Na}$ função CLASSIFICAR, o manual em IO possui a opção de cosselecionar com o Modo 20 e com o Tema 10/41, o Processo 39/37. Essas opções não ocorrem no manual em PO.

Com relação à função INTRODUZIR, a confluência entre Tema 41, o Modo 20 e o Processo 29 não ocorre no manual em PO.

Comparando com base no manual em PO, na função COMANDAR, não existe no IO a cosseleção do Tema 41 com o Modo 11 e o Processo 59.

$\mathrm{Na}$ função INTRODUZIR, a opção composta pelo Processo 41 confluindo com o Modo 20 e o Tema 10/41 não existe no manual em IO.

Com relação às demais cosseleções gramaticais que não foram mencionadas anteriormente, o Quadro 5 a seguir apresenta as suas ocorrências com suas respectivas funções semânticas. 
Quadro 5 - Significados multilíngues do manual de instrução em inglês e português brasileiro

\begin{tabular}{lclc}
\hline \multicolumn{3}{c}{ Cosseleções Gramaticais } & Funções Semânticas \\
\hline Trans. & Modo & Tema & \\
$14 \backslash 16$ & 20 & $10 \backslash 41$ & \\
59 & 20 & 41 & EXPLICAR \\
$14 \backslash 16$ & 11 & $10 \backslash 41$ & \\
59 & 11 & 10 & COMANDAR \\
$33 \backslash 32$ & 20 & $10 \backslash 41$ & CLASSIFICAR \\
29 & 20 & 10 & INTRODUZIR \\
\hline
\end{tabular}

O Quadro 5 aponta as categorias gramaticais e as funções semânticas comuns entre os manuais em inglês e em português brasileiro original. Nesse sentido, todas as classes apresentadas nesse quadro representam a correspondência formal entre as duas línguas para o manual de instrução, ou os seus significados multilíngues (MATTHIESSEN et al., 2008).

\section{Perfilação gramatical sistêmica do corpus combinado}

Com base nos resultados das frequências relativas a cada categoria gramatical analisada no corpus combinado deste trabalho e por meio da análise das funções semânticas e as ondas de informação, é possível apresentar um perfil gramatical sistêmico que constitui um modelo da variação linguística multilíngue para os quatro manuais de instrução.

O modelo não é nenhum texto específico, criado por usuários do português brasileiro. Ele é, por outro lado, uma generalização das probabilidades maiores de escolhas dos sistemas gramaticais para o corpus de manuais. Assim, o perfil a seguir não se refere especificamente a qualquer texto em particular, mas propõe uma previsão para o comportamento da gramática oracional do português brasileiro para os textos caracterizados como manual de instrução.

O Quadro 6 apresenta o perfil sistêmico para o corpus combinado no par linguístico inglês e português brasileiro deste artigo. 
Quadro 6 - Perfilação sistêmica do corpus combinado de manuais de instrução

(Continua)

\begin{tabular}{|c|c|c|c|c|c|c|c|c|c|}
\hline \multicolumn{10}{|c|}{ PERFIL SISTÊMICO DO CORPUS COMBINADO } \\
\hline $\begin{array}{l}\text { Número da } \\
\text { oração }\end{array}$ & $\begin{array}{l}\text { Trans. } \\
\text { (x) }\end{array}$ & $\begin{array}{l}\text { Modo } \\
\text { (y) }\end{array}$ & $\begin{array}{c}\text { Tema } \\
\text { (z) }\end{array}$ & $\begin{array}{c}\text { Ondas } \\
\text { de } \\
\text { infor- } \\
\text { mação }\end{array}$ & $\begin{array}{l}\text { Número da } \\
\text { oração }\end{array}$ & $\begin{array}{c}\text { Trans. } \\
\text { (x) }\end{array}$ & $\begin{array}{l}\text { Modo } \\
\text { (y) }\end{array}$ & $\begin{array}{l}\mathrm{Te} \\
\mathrm{ma} \\
(\mathrm{z})\end{array}$ & $\begin{array}{l}\text { Ondas } \\
\text { de infor- } \\
\text { mação }\end{array}$ \\
\hline FRANK_01 & 14 & 20 & 10 & $1^{\mathrm{a}}$ & FRANK_01 & \multicolumn{2}{|c|}{ EXPLICAR } & & \multirow[t]{5}{*}{$1^{\mathrm{a}}$} \\
\hline FRANK_02 & 14 & 20 & 10 & & FRANK_02 & \multicolumn{2}{|c|}{ EXPLICAR } & & \\
\hline FRANK 03 & 33 & 20 & 41 & & FRANK 03 & \multicolumn{2}{|c|}{ CLASSIFICAR } & & \\
\hline FRANK 04 & 14 & 11 & 10 & & FRANK 04 & \multicolumn{2}{|c|}{ COMANDAR } & & \\
\hline FRANK_05 & 14 & 20 & 10 & & FRANK_05 & \multicolumn{2}{|c|}{ EXPLICAR } & & \\
\hline FRANK_06 & 14 & 20 & 41 & $2^{\mathrm{a}}$ & FRANK_06 & \multicolumn{2}{|c|}{ EXPLICAR } & & \multirow[t]{7}{*}{$2^{\mathrm{a}}$} \\
\hline FRANK_07 & 32 & 20 & 10 & & FRANK_07 & \multicolumn{2}{|c|}{ CLASSIFICAR } & & \\
\hline FRANK_08 & 14 & 20 & 10 & & FRANK_08 & \multicolumn{2}{|c|}{ EXPLICAR } & & \\
\hline FRANK_09 & 59 & 20 & 41 & & FRANK_09 & \multicolumn{2}{|c|}{ EXPLICAR } & & \\
\hline FRANK 10 & 14 & 20 & 10 & & FRANK ${ }^{-} 10$ & \multicolumn{2}{|c|}{ EXPLICAR } & & \\
\hline FRANK 11 & 14 & 11 & 10 & & FRANK 11 & \multicolumn{2}{|c|}{ COMANDAR } & & \\
\hline FRANK 12 & 14 & 11 & 10 & & FRANK 12 & \multicolumn{2}{|c|}{ COMANDAR } & & \\
\hline FRANK_13 & 59 & 11 & 41 & $3^{\mathrm{a}}$ & FRANK_13 & \multicolumn{2}{|c|}{ COMANDAR } & & \multirow[t]{9}{*}{$3^{a}$} \\
\hline FRANK_14 & 41 & 20 & 10 & & FRANK_14 & \multicolumn{2}{|c|}{ INTRODUZIR } & & \\
\hline FRANK_15 & 14 & 11 & 10 & & FRANK_15 & \multicolumn{2}{|c|}{ COMANDAR } & & \\
\hline FRANK_16 & 14 & 11 & 10 & & FRANK_16 & \multicolumn{2}{|c|}{ COMANDAR } & & \\
\hline FRANK 17 & 14 & 20 & 10 & & FRANK 17 & \multicolumn{2}{|c|}{ EXPLICAR } & & \\
\hline FRANK 18 & 33 & 20 & 41 & & FRANK 18 & \multicolumn{2}{|c|}{ CLASSIFICAR } & & \\
\hline FRANK_19 & 14 & 20 & 10 & & FRANK_19 & \multicolumn{2}{|c|}{ EXPLICAR } & & \\
\hline FRANK_20 & 32 & 20 & 41 & & FRANK_20 & \multicolumn{2}{|c|}{ CLASSIFICAR } & & \\
\hline FRANK_21 & 14 & 20 & 41 & & FRANK_21 & \multicolumn{2}{|c|}{ EXPLICAR } & & \\
\hline FRANK_22 & 16 & 20 & 10 & $4^{a}$ & FRANK_22 & EXPI & CAR & & $4^{\mathrm{a}}$ \\
\hline FRANK_23 & 29 & 20 & 10 & & FRANK_23 & INTRC & UZIR & & \\
\hline FRANK 24 & 14 & 11 & 10 & & FRANK 24 & $\mathrm{COM}$ & IDAR & & \\
\hline FRANK 25 & 14 & 11 & 10 & & FRANK 25 & $\mathrm{COM}$ & IDAR & & \\
\hline FRANK_26 & 14 & 11 & 10 & $5^{\mathrm{a}}$ & FRANK_26 & $\mathrm{COM}$ & IDAR & & $5^{\mathrm{a}}$ \\
\hline FRANK_27 & 14 & 11 & 10 & & FRANK_27 & $\mathrm{COM}$ & JDAR & & \\
\hline FRANK_28 & 14 & 11 & 41 & & FRANK_28 & $\mathrm{COM}$ & IDAR & & \\
\hline FRANK_29 & 14 & 11 & 10 & & FRANK_29 & $\mathrm{COM}$ & IDAR & & \\
\hline FRANK_30 & 11 & 11 & 10 & & FRANK_30 & $\mathrm{COM}$ & IDAR & & \\
\hline FRANK_31 & 14 & 11 & 10 & & FRANK_31 & $\mathrm{COM}$ & IDAR & & \\
\hline FRANK 32 & 14 & 11 & 41 & & FRANK 32 & $\mathrm{COM}$ & IDAR & & \\
\hline FRANK_33 & 14 & 11 & 41 & & FRANK_33 & $\mathrm{COM}$ & IDAR & & \\
\hline FRANK_34 & 14 & 11 & 10 & & FRANK_34 & $\mathrm{COM}$ & IDAR & & \\
\hline FRANK_35 & 14 & 11 & 41 & & FRANK_35 & EXPI & CAR & & \\
\hline FRANK_36 & 16 & 20 & 41 & $6^{\mathrm{a}}$ & FRANK_36 & $\mathrm{COM}$ & IDAR & & $6^{\mathrm{a}}$ \\
\hline FRANK 37 & 14 & 20 & 41 & & FRANK 37 & EXPI & CAR & & \\
\hline
\end{tabular}


Quadro 6 - Perfilação sistêmica do corpus combinado de manuais de instrução

(Conclusão)

\begin{tabular}{|c|c|c|c|c|c|c|c|c|c|}
\hline \multicolumn{10}{|c|}{ PERFIL SISTÊMICO DO CORPUS COMBINADO } \\
\hline $\begin{array}{l}\text { Número da } \\
\text { oração }\end{array}$ & $\begin{array}{l}\text { Trans. } \\
\text { (x) }\end{array}$ & $\begin{array}{l}\text { Modo } \\
\text { (y) }\end{array}$ & $\begin{array}{c}\text { Tema } \\
\text { (z) }\end{array}$ & $\begin{array}{l}\text { Ondas } \\
\text { de } \\
\text { infor- } \\
\text { mação }\end{array}$ & $\begin{array}{l}\text { Número da } \\
\text { oração }\end{array}$ & $\begin{array}{c}\text { Trans. } \\
\text { (x) }\end{array}$ & $\begin{array}{l}\text { Modo } \\
\text { (y) }\end{array}$ & $\begin{array}{l}\mathrm{Te} \\
\mathrm{ma} \\
(\mathrm{z})\end{array}$ & $\begin{array}{l}\text { Ondas } \\
\text { de infor- } \\
\text { mação }\end{array}$ \\
\hline FRANK 38 & 59 & 11 & 10 & $7^{a}$ & FRANK 38 & $\mathrm{COM} A$ & DAR & & $7^{\mathrm{a}}$ \\
\hline FRANK 39 & 37 & 20 & 10 & & FRANK 39 & \multicolumn{3}{|c|}{ CLASSIFICAR } & \\
\hline FRANK 40 & 16 & 20 & 10 & & FRANK 40 & \multicolumn{3}{|c|}{ EXPLICAR } & \\
\hline FRANK 41 & 37 & 20 & 10 & & FRANK 41 & \multicolumn{3}{|c|}{ CLASSIFICAR } & \\
\hline FRANK_42 & 14 & 11 & 10 & & FRANK 42 & \multicolumn{3}{|c|}{ COMANDAR } & \\
\hline FRANK 43 & 14 & 20 & 41 & & FRANK 43 & \multicolumn{3}{|c|}{ EXPLICAR } & \\
\hline FRANK 44 & 14 & 20 & 10 & $8^{a}$ & FRANK 44 & & $8^{\mathrm{a}}$ \\
\hline FRANK ${ }^{-} 45$ & 33 & 20 & 10 & & FRANK ${ }^{-} 45$ & \multirow{2}{*}{\multicolumn{3}{|c|}{ CLASSIFICAR }} & \\
\hline FRANK 46 & 59 & 11 & 10 & $9^{a}$ & FRANK 46 & & & COMANDAR & $9^{a}$ \\
\hline FRANK 47 & 54 & 11 & 10 & $10^{\mathrm{a}}$ & FRANK 47 & \multicolumn{2}{|c|}{ COMANDAR } & & $10^{\mathrm{a}}$ \\
\hline FRANK ${ }^{-} 48$ & 14 & 11 & 41 & & FRANK $^{-} 48$ & \multicolumn{2}{|c|}{ COMANDAR } & & \\
\hline FRANK ${ }^{-} 49$ & 14 & 20 & 10 & & FRANK ${ }^{-} 49$ & \multicolumn{2}{|c|}{ EXPLICAR } & & \\
\hline FRANK 50 & 14 & 20 & 10 & & FRANK 50 & \multicolumn{2}{|c|}{ EXPLICAR } & & \\
\hline FRANK_51 & 14 & 11 & 10 & $11^{\mathrm{a}}$ & FRANK_51 & \multicolumn{2}{|c|}{ COMANDAR } & & $11^{\mathrm{a}}$ \\
\hline FRANK 52 & 14 & 11 & 41 & & FRANK 52 & \multicolumn{2}{|c|}{ COMANDAR } & & \\
\hline FRANK 53 & 14 & 11 & 10 & & FRANK 53 & \multicolumn{2}{|c|}{ COMANDAR } & & \\
\hline FRANK 54 & 14 & 11 & 10 & & FRANK 54 & \multicolumn{2}{|c|}{ COMANDAR } & & \\
\hline FRANK 55 & 14 & 11 & 41 & $12^{\mathrm{a}}$ & FRANK 55 & \multirow{2}{*}{\multicolumn{2}{|c|}{$\begin{array}{l}\text { COMANDAR } \\
\text { COMANDAR }\end{array}$}} & & $12^{\mathrm{a}}$ \\
\hline FRANK_56 & 14 & 11 & 10 & & FRANK_56 & & & & \\
\hline FRANK 57 & 14 & 11 & 10 & & FRANK 57 & \multicolumn{2}{|c|}{ COMANDAR } & & \\
\hline FRANK 58 & 14 & 11 & 10 & & FRANK 58 & \multicolumn{2}{|c|}{ COMANDAR } & & \\
\hline FRANK 59 & 29 & 20 & 10 & $13^{\mathrm{a}}$ & FRANK 59 & \multicolumn{2}{|c|}{ INTRODUZIR } & & $13^{\mathrm{a}}$ \\
\hline FRANK 60 & 33 & 11 & 41 & $14^{\mathrm{a}}$ & FRANK 60 & \multicolumn{2}{|c|}{ COMANDAR } & & $14^{\mathrm{a}}$ \\
\hline FRANK 61 & 14 & 11 & 41 & & FRANK 61 & \multicolumn{2}{|c|}{ COMANDAR } & & \\
\hline FRANK 62 & 14 & 20 & 10 & $15^{\mathrm{a}}$ & FRANK 62 & \multicolumn{2}{|c|}{ EXPLICAR } & & $15^{\mathrm{a}}$ \\
\hline FRANK 63 & 14 & 11 & 41 & & FRANK 63 & \multicolumn{2}{|c|}{ COMANDAR } & & \\
\hline FRANK 64 & 14 & 11 & 10 & & FRANK 64 & COM & IDAR & & \\
\hline FRANK 65 & 14 & 11 & 10 & & FRANK 65 & COM & IDAR & & \\
\hline FRANK_66 & 14 & 20 & 41 & & FRANK_66 & EXPI & CAR & & \\
\hline FRANK_67 & 14 & 20 & 10 & $16^{\mathrm{a}}$ & FRANK_67 & EXPI & CAR & & $16^{\mathrm{a}}$ \\
\hline FRANK 68 & 32 & 20 & 10 & & FRANK 68 & CLASS & ICAR & & \\
\hline FRANK 69 & 14 & 11 & 10 & & FRANK 69 & $\mathrm{COM} A$ & IDAR & & \\
\hline FRANK_70 & 16 & 11 & 10 & & FRANK_70 & $\mathrm{COM}$ & IDAR & & \\
\hline FRANK 71 & 59 & 20 & 10 & & FRANK 71 & EXPI & CAR & & \\
\hline FRANK_72 & 14 & 20 & 10 & & FRANK_72 & EXPI & CAR & & \\
\hline FRANK 73 & 14 & 20 & 10 & & FRANK 73 & EXPI & CAR & & \\
\hline FRANK 74 & 14 & 20 & 10 & & FRANK 74 & EXPI & CAR & & \\
\hline
\end{tabular}

Esse modelo, também é chamado de Frankenstein por representar o que é mais frequente dos quatro manuais de instrução; é composto por 74 mensagens e 16 ondas de informação. A primeira seção desse quadro aponta as cosseleções gramaticais de cada mensagem; a segunda, mostra 
o significado semântico resultante da confluência das classes da TRANSITIVIDADE, MODO e TEMA.

O Frankenstein não é considerado um manual, e sim um modelo criado para compreender como são realizadas pelo falante as escolhas gramaticais e, consequentemente, semânticas (HALLIDAY et al., 1964), a fim de construir o significado do registro: manual de instrução. Para exemplificar o Frankenstein, o Quadro 7 mostra a sua primeira onda de informação, com exemplos do corpus combinado. Os exemplos seguem a descrição de cada oração exibida no Quadro 6.

Quadro 7- O Frankenstein com exemplos do corpus

\begin{tabular}{|c|c|c|}
\hline ORAÇÃO & EXEMPLOS & $\begin{array}{l}\text { TEXTO } \\
\text { FONTE }\end{array}$ \\
\hline FRANK 01 & Você adquiriu a panela de pressão solar Tramontina & $\mathrm{PO}$ \\
\hline FRANK 02 & This guide shows you what's on your Mac & IO \\
\hline FRANK_03 & $\begin{array}{l}\text { para que eles estejam prontos quando você utilizá-los pela } \\
\text { primeira vez. }\end{array}$ & PT \\
\hline FRANK_ 04 & Clean the pressure regulator valve ( 8 ) & IT \\
\hline FRANK_05 & $\begin{array}{l}\text { Este dispositivo impede a abertura da tampa enquanto } \\
\text { houver pressão no interior da panela. }\end{array}$ & PO \\
\hline
\end{tabular}

O Quadro 7 mostra o Frankenstein criado com partes distintas dos manuais do corpus. Apesar de ele não apresentar os mesmos participantes nas orações e de não haver conectividade entre um termo e outro do ponto de vista lexical, esse modelo está gramaticalmente correto, no sentido de que ele representa ideacionalmente, encena interpessoalmente e constrói textualmente a primeira onda de informação do corpus, que trata principalmente da identificação do objeto abordado pelo manual. Ademais, faz isso com base nas probabilidades maiores de escolha de funções dos sistemas envolvidos para cada oração.

Por conseguinte, a comparação realizada neste trabalho também apresenta o ambiente multilíngue comum dos quatro textos no par linguístico inglês / português brasileiro com suas respectivas traduções. A Figura 2 mostra o sistema multilíngue da realização semântica pautada em escolhas gramaticais do corpus combinado de manuais. 
Figura 2 - Sistema Multilíngue da realização semântica pautada em escolhas gramaticais do corpus combinado de manuais

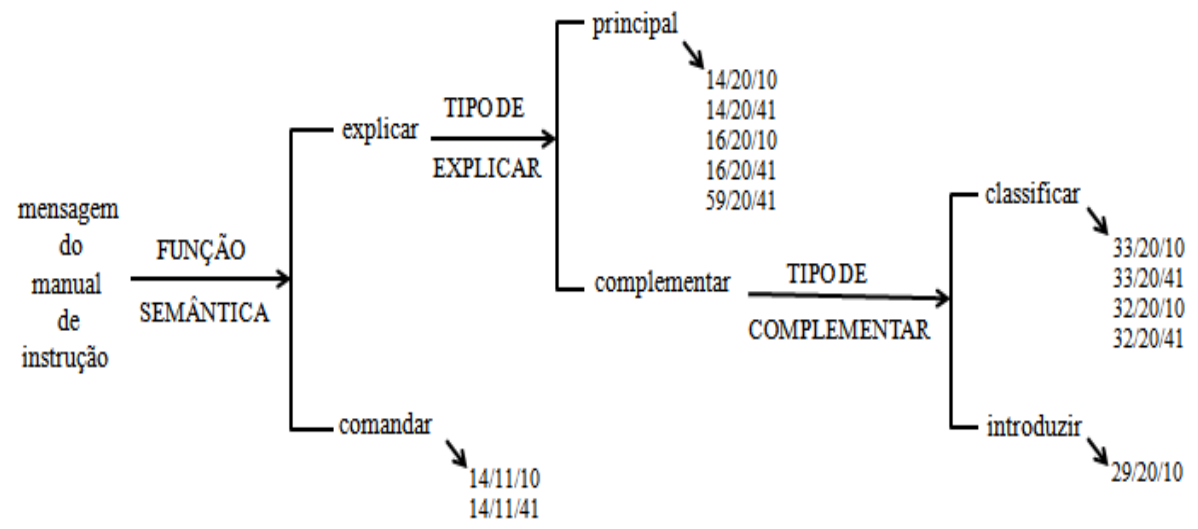

Fonte: a autora.

A Figura 2 representa a construção de significados partilhados entre as mensagens do corpus. Dessa forma, ela apresenta o que é o manual de instrução no ambiente multilíngue.

Nesse sistema, a condição de entrada é a mensagem do manual de instrução. No primeiro nível de delicadeza do sistema, a mensagem do manual pode apresentar a função semântica 'explicar' ou 'comandar'. Em relação ao TIPO DE EXPLICAR, ele pode ser 'principal' ou 'complementar'. Caso a seleção seja por 'complementar', apresentam-se mais duas classes: 'classificar' ou 'introduzir'. As setas que seguem nas classes apontam as opções gramaticais de cada uma.

\section{Conclusões}

Tendo como foco principal apresentar como o arcabouço da Linguística de Corpus com a Linguística Sistêmico-Funcional permite, pela perfilação gramatical, analisar, comparar e encontrar padrões linguísticos tanto para o registro quanto para a tradução técnica, este artigo demonstrou os padrões gramaticais e semânticos de um corpus combinado: manual de instrução. 
Mediante a análise do corpus e os padrões encontrados (BIBER, 2010), os resultados apontaram que o manual de instrução é uma macroonda de significado que capacita o falante a desenvolver uma atividade. Mais especificamente, esse registro tem por objetivo instruir o falante e não regular o seu comportamento. Para tanto, o manual utiliza quatro funções semânticas para significar, a saber, a função EXPLICAR, que é composta pelas funções CLASSIFICAR e INTRODUZIR, e a função COMANDAR. Nesse sentido, o manual é uma sequência de explicações intercaladas com comandos em que o objeto do manual e o leitor são negociados, a fim de construir a macro função capacitar.

Os resultados das análises apontaram semelhanças e diferenças entre as línguas, como a função semântica CONVIDAR e sua realização gramatical que ocorreu somente no manual em inglês original, ou a cosseleção na função INTRODUZIR com o Processo: Verbal que ocorreu somente no manual em português original.

Com relação à tradução técnica, cumpre destacar que os manuais traduzidos do corpus apresentaram construções gramaticais e semânticas do texto fonte, do texto original da língua alvo e construções que não apareceram nesses dois textos, por exemplo, a cosseleção de 33/11/10 ou 41 na função COMANDAR do manual em inglês traduzido que não ocorre no manual em inglês original. Portanto, a tradução no corpus de manuais é um texto híbrido e multilíngue que, para significar, utiliza-se do pareamento do texto fonte, da língua alvo e de novos significados.

Ao final, o perfil gramatical sistêmico do corpus (o Frankenstein) apresentou a confluência de significados dos manuais desta pesquisa, mostrando, assim, o que é o manual com todos os significados mais frequentes desse ambiente multilíngue.

Por fim, cabe ressaltar que, para pesquisas futuras, este trabalho contribui como um método de pesquisa que amplia a análise de corpus (VIANA, 2011), utilizando a perfilação sistêmica e a comparação linguística. Quanto ao produto final desta pesquisa, o modelo da variação linguística contribui com o estudo dos padrões linguísticos do registro: manual de instrução (BIBER, 2010). E, com relação à tradução, os procedimentos aqui apresentados mostram uma possibilidade de analisar traduções técnicas por meio de um corpus combinado (STEINER, 2001; NUNES, 2013), ampliando, assim, os estudos sobre tradução. 


\section{Referências}

AZENHA, J. Tradução técnica, condicionantes culturais e os limites da responsabilidade do tradutor. In: CONGRESSO BRASILEIRO DE LINGÜÍSTICA APLICADA, 4, 1995, Campinas. Anais... UNICAMP, Campinas, SP, 1995. p. 137-149.

APPLE - Macbook Pro retina 13 inch. Disponível em: $<$ http://manuals.info.apple.com/MANUALS/1000/MA1663/en_US/macb ook_pro_retina-13-inch-late-2013_qs.pdf $>$. Acesso em: 22 jan. 2014.

BERBER SARDINHA, T. Linguística de corpus: histórico e problemática. D.E.L.T.A., v. 16, n. 2, p. 323-367, 2000.

BERBER SARDINHA, T. Corpora eletrônicos na pesquisa em tradução. São Paulo: LAEL, PUC/SP, 2002. p. 15-59.

BIBER, D. M. Methodological issues regarding corpus-based analyses of linguistic variation. Literary and Linguistic Computing, Oxford, v. 5, n. 4, p. 257-269, 1990. DOI: $<$ http://dx.doi.org/10.1093/1lc/5.4.257>

. What can a corpus tell us about registers and genres? In: O'KEEFFE, A.; McCARTHY, M. (Ed.). The Routledge Handbook of corpus Linguistics. London, New York: Routledge, 2010. p. 241-254.

BIBER, D.; CONRAD, S.; CORTES, V. If You Look at...: Lexical Bundles in University Teaching and Textbooks. Applied Linguistics, Oxford, n. 25(3), p. 371-405, 2004.

CATFORD, J. A linguistic theory of translation: an essay in applied linguistics. London: Oxford University, 1965.

FIGUEREDO, G. Introdução ao perfil metafuncional do português brasileiro: contribuições para os estudos multilíngues. 2011. 385 f. Tese (Doutorado em Linguística Aplicada) - Programa de Pós-Graduação em Estudos Linguísticos, Faculdade de Letras, Universidade Federal de Minas Gerais, Belo Horizonte, 2011.

FIGUEREDO, G. Uma metodologia de perfilação gramatical sistêmica baseada em corpus. Letras \& Letras, Uberlândia, v. 30, n. 2, p. 17-45, jul./dec. 2014. DOI: $<$ http://dx.doi.org/10.14393/LL60-v30n2a2014-2> 
FIGUEREDO, G. Um estudo do conjunto multilíngue interpessoal português brasileiro / inglês subsidiado pelos estudos da tradução e pela linguística sistêmico-functional. Cad. Trad., Florianópolis, v. 35, n. 1, p. 139-166, 2015.

HALLIDAY, M. A. K. Language as social semiotic: the social interpretation of language and meaning. London \& Baltimore: Edward Arnold\& University Park Press, 1978.

. The construction of knowledge and value in the grammar of scientific discourse, with reference to Charles Darwing's the origin of species. In: COULTHARD, M. (Ed.). Advances in written text analysis. London and New York: Routledge, 1994.

. On grammar. London: Continuum, 2002. (The collected works of M. A. K. Halliday, v. 1).

. Meaning as choice. In: FONTAINE, L.; BARTLETT, T.; O'GRADY, G. (Ed.). Systemic Functional Linguistics: exploring choice. New York: Cambridge University Press, 2013. p. 15-36.

DOI: $<$ http://dx.doi.org/10.1017/CBO9781139583077>

HALLIDAY, M. A. K.; MCINTOSH, A.; STREVENS, P. The linguist sciences and language teaching. London: Longman, 1964.

HALLIDAY, M. A. K.; MATTHIESSEN, M. I. M. Introduction to Functional Grammar. London and New York: Routledge, 2014.

KENNING, M. 2010. What are parallel and comparable corpora and how can we use them? In: O'KEEFFE, A.; McCARTHY, M. (Ed.). The Routledge Handbook of corpus Linguistics. London, New York:

Routledge, 2010. p. 487-500.

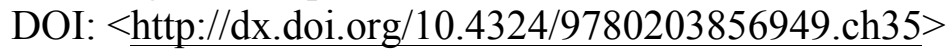

KRZESZOWSKI, T. Contrasting languages. The scope of contrastive linguistics. Berlin: De Gruyter, 1990.

DOI: $<$ http://dx.doi.org/10.1515/9783110860146>

LEMKE, J. Discourse, Dynamics, and Social Change. Cultural Dynamics, v. 6, n. 1, p. 243-275, 1993. 
DOI: $<$ http://dx.doi.org/10.1177/092137409300600107> .

MARTIN, J. R. Systemic functional grammar: a next step into the theory - axial relations. Beijing: Higher Education Press, 2013.

MARTIN, J. R.; ROSE, David. Working with discourse: meaning beyond the clause. 2 ed. London: Continuum, 2007.

MATTHIESSEN, C. The environments of translation. In: STEINER, E. YALLOP, C. (Ed.). Exploring translation and multilingual text production beyond content. Berlin, New York: Mouton de Gruyer, 2001. p. 41-124. DOI: <http://dx.doi.org/10.1515/9783110866193.41>

MATTHIESSEN, C.; TERUYA, K.; WU, C. Multilingual studies as a multidimensional space of interconnected language studies. In: WEBSTER, J. (Ed.). Meaning in Context: implementing intelligent applications of language studies. London, New York: Continuum, 2008. p. 146-220.

NUNES, L. P. Relações coesivas e estruturais em corpus combinado: uma análise de conjunções em textos originais e traduzidos. Belo Horizonte: Universidade Federal de Minas Gerais, 2013. (Trabalho de qualificação para o doutorado). (Mimeo)

O'DONNELL, M. The UAM CorpusTool: software for corpus annotation and exploration. In: BRETONES CALLEJAS, C. M. et al. (Ed.). Applied linguistics now: understanding language and mind. Almería: Universidad de Almería, 2008. p. 1433-1447.

SINCLAIR, J. Corpus evidence in language description. In: WICHMANN, A. et al. (Ed.). Teaching and language corpora. London: Longman, 1997. p. 27-39.

STEINER, E. Translations English - German: investigating the relative importance of systemic contrasts and of the text-type "translation". SPRIKreports, n. 7, p. 1-49, Oct. 2001. Disponível em: $<$ http:/hf.ulo.no/ilos/forkning/prosjekter/sprik/docs/pdf/steiner.pdf $>$.

TRAMONTINA. Tramontina Solar. Disponível em: $<$ http://suipnovo.tecnologia.ws/public/upload/product/62516223/625162 22FLM001.pdf>. Acesso em: 22 jan. 2014. 
VIANA, V. Linguística de corpus: conceitos, técnicas e análises. In: VIANA, V.; TAGNIN, S. (Org.). Corpora no ensino de línguas estrangeiras. São Paulo: HUB Editorial, 2011. p. 25-95.

URE, J. Text types classified by situational factors. Atlanta: MS, 1989. 